\title{
RNA interference therapeutics targeting angiotensinogen ameliorate preeclamptic phenotype in rodent models
}

\author{
Nadine Haase, ${ }^{1,2,3,4,5}$ Donald J. Foster, ${ }^{6}$ Mark W. Cunningham, ${ }^{7}$ Julia Bercher, ${ }^{3}$ Tuyen Nguyen, ${ }^{6}$ Svetlana Shulga-Morskaya, ${ }^{6}$ \\ Stuart Milstein, ${ }^{6}$ Sarfraz Shaikh, ${ }^{6}$ Jeff Rollins, ${ }^{6}$ Michaela Golic, ${ }^{1,2,3,5}$ Florian Herse, ${ }^{1,2,3,5}$ Kristin Kräker, ${ }^{1,2,3,4,5}$ Ivo Bendix, ${ }^{8}$ \\ Meray Serdar, ${ }^{8}$ Hanna Napieczynska, ${ }^{1}$ Arnd Heuser, ${ }^{1}$ Alexandra Gellhaus, ${ }^{9}$ Kristin Thiele, ${ }^{10}$ Gerd Wallukat, ${ }^{3}$ \\ Dominik N. Müller, ,2,3,4,5 Babbette LaMarca, ${ }^{7,11}$ and Ralf Dechend ${ }^{1,2,3,5,12}$ \\ ${ }^{1}$ Max-Delbrück Center for Molecular Medicine in the Helmholtz Association, Berlin, Germany. ${ }^{2}$ Charité - Universitätsmedizin Berlin, Berlin Germany. ${ }^{3}$ Experimental and Clinical Research Center, Berlin, \\ Germany. ${ }^{4}$ DZHK (German Centre for Cardiovascular Research), Berlin, Germany. ${ }^{5}$ Berlin Institute of Health (BIH), Berlin, Germany. ${ }^{6}$ Alnylam Pharmaceuticals, Cambridge, Massachusetts, USA. ${ }^{7}$ Department \\ of Pharmacology and Toxicology, University of Mississippi Medical Center, Jackson, Mississippi, USA. ${ }^{8}$ Department of Pediatrics I Neonatology and Experimental Perinatal Neurosciences and ${ }^{9}$ Department \\ of Gynecology and Obstetrics, University Hospital Essen, University Duisburg-Essen, Essen, Germany. ${ }^{10}$ Department of Experimental Feto-Maternal Medicine, University Medical Center Hamburg-Eppendorf, \\ Hamburg, Germany. "Department of Obstetrics and Gynecology, University of Mississippi Medical Center, Jackson, Mississippi, USA. ${ }^{12} \mathrm{HELIOS}$-Klinikum, Berlin, Germany.
}

\begin{abstract}
Preeclampsia, with the hallmark features of new-onset hypertension and proteinuria after $\mathbf{2 0}$ weeks of gestation, is a major cause of fetal and maternal morbidity and mortality. Studies have demonstrated a role for the renin-angiotensin system (RAS) in its pathogenesis; however, small-molecule RAS blockers are contraindicated because of fetal toxicity. We evaluated whether siRNA targeting maternal hepatic angiotensinogen (Agt) could ameliorate symptoms of preeclampsia without adverse placental or fetal effects in 2 rodent models. The first model used a cross of females expressing human Agt with males expressing human renin, resulting in upregulation of the circulating and uteroplacental RAS. The second model induced ischemia/reperfusion injury and subsequent local and systemic inflammation by surgically reducing placental blood flow midgestation (reduced uterine perfusion pressure [RUPP]). These models featured hypertension, proteinuria, and fetal growth restriction, with altered biomarkers. siRNA treatment ameliorated the preeclamptic phenotype in both models, reduced blood pressure, and improved intrauterine growth restriction, with no observed deleterious effects on the fetus. Treatment also improved the angiogenic balance and proteinuria in the transgenic model, and it reduced angiotensin receptor activating antibodies in both. Thus, an RNAi therapeutic targeting Agt ameliorated the clinical sequelae and improved fetal outcomes in 2 rodent models of preeclampsia.
\end{abstract}

\section{Introduction}

As one of the leading causes of maternal mortality worldwide, hypertensive disorders of pregnancy remain inadequately treated (1). Preeclampsia, a subset of such disorders, occurs in $5 \%$ to $8 \%$ of pregnancies, threatening to continue to fulminant eclampsia (1). Preeclampsia is characterized by new-onset maternal hypertension $(>140 / 90 \mathrm{mmHg}$ ) after the 20th week of gestation accompanied by proteinuria (>300 $\mathrm{mg} / \mathrm{L}$ protein in a 24 -hour urine collection) or in association with thrombocytopenia, impaired liver function,

Conflict of interest: DF, TN, SSM, SM, SS, and JR are employees of Alnylam Pharmaceuticals Inc. and own Alnylam stock. Alnylam Pharmaceuticals Inc. is the owner of patent applications and patents directed to siRNA targeting angiotensinogen, and the sponsor of a clinical trial (NCT03934307). Alnylam is the applicant on PCT Publications W02015/179724 and WO2019/222166 related to AGT siRNA and applications and patents based thereon.

Copyright: () 2020, American Society for Clinical Investigation. Submitted: December 26, 2017; Accepted: February 13, 2020; Published: April 27, 2020.

Reference information: J Clin Invest. 2020;130(6):2928-2942. https://doi.org/10.1172/JCI99417. development of renal insufficiency, pulmonary edema, or newonset cerebral or visual disturbances (2). Furthermore, it is associated with negative fetal consequences, with fetal growth restriction (FGR) a common outcome. Newborns with FGR have structural and metabolic abnormalities that compromise their development (3). Moreover, preeclampsia is associated with increased long-term cardiovascular risk for both the mother and the child $(3,4)$.

Preeclampsia is distinct in its complex pathophysiology, and the molecular mechanisms are not understood. Poor placentation, as well as maternal risk factors leading to placental dysfunction, is involved in the pathogenesis (1). Many factors have been identified as having an involvement in the progression of preeclampsia, including dysfunction of the renin-angiotensin system (RAS) (5).

As the primary effector of the RAS, angiotensin II is a peptide hormone with vasoconstrictive and inflammatory properties, and forms a major component of blood pressure homeostasis (6). Angiotensin II is produced by the successive actions of renin and angiotensin-converting enzyme (ACE) on angiotensinogen (AGT) and angiotensin I, respectively. AGT M235T and other mutations have been associated with increased AGT plasma levels (7), ele- 
vated systolic and diastolic blood pressure, and hypertension (8). Conversely, lowering plasma AGT levels via targeting of hepatic Agt expression with small interfering RNA (siRNA) or antisense oligonucleotides in a rat model of hypertension lowers blood pressure (9). Similarly, liver-specific knockout of Agt in mice confirms that liver is the primary source of circulating AGT (10). Although Agt mRNA is present in other organs, such as kidney and adipose, it appears that the functional angiotensin II stems from circulating (liver-derived) AGT (11).

General dysfunction of the RAS in preeclampsia was first described by Gant et al., demonstrating hypersensitivity to angiotensin II in women before the onset of hypertension during pregnancy (12). Subsequent studies have identified elevated levels of the type 1 angiotensin receptor (AT1) (13), heterodimerization of AT1 with bradykinin receptor B2 (14), and agonistic AT1 autoantibodies (AT1-AA) (15). AGT has also been linked to preeclampsia in multiple clinical studies. The AGT M235T mutation, which is associated with higher plasma levels of AGT, is associated with preeclampsia (16). An oxidized form of AGT was also observed to be more prevalent in preeclamptic women than in normotensive maternal controls and to be associated with a 4 -fold increase in catalytic release of angiotensin I in the presence of prorenin receptor (16). Similarly, a mutation in the renin cleavage site (L10F) was identified in a patient with preeclampsia. The mutation enabled a 2-fold increase in the catalytic efficiency of cleavage by renin and a greater than 2 -fold increase in the rate of angiotensin II formation by ACE (17).

How such perturbations may interact is not fully understood, but it is known that AT1-AA can induce angiotensin sensitivity (18), possibly through impaired internalization of AT1 (18). Consistent with this, administration of an ACE inhibitor or angiotensin II receptor blocker reduces blood pressure in both the reduced uterine perfusion pressure (RUPP) model (where AT1-AA are present) and the AT1-AA infusion model $(19,20)$. However, ACE inhibitor use is associated with risk of fetal death, FGR, and renal abnormalities $(21,22)$. Moreover, there is a negative relationship between use of non-RAS-blocking antihypertensive drugs and birth weight (23). In the CHIPS trial, a lower blood pressure target was associated with a trend to increased risk of small-for-gestational-age (SGA) birth (CI, 0.44-1.0) (24). Thus, a fetal-sparing antihypertensive, in addition to mitigating the potential role of the RAS in preeclampsia, may be beneficial.

A cross of rats or mice bearing human AGT $(h A g t)$ and human renin (hRen) has been demonstrated to reproduce much of the pathology observed in preeclampsia, including hypertension, proteinuria, FGR, and elevated factors such as AT1 agonistic autoantibodies $(15,25,26)$. As both tissue and circulating reninangiotensin systems have been implicated, including uteroplacenta (27), it is unclear to what extent these systems contribute to pathology in this model $(26,27)$.

Another animal model of preeclampsia is the reduced uterine perfusion pressure (RUPP) model. In this model, surgically induced placental ischemia gives way to hypertension, systemic inflammation, oxidative stress, and FGR (28). As with the transgenic model above, the RUPP model is associated with alterations in the RAS, including agonistic autoantibodies. Although the application of an ACE inhibitor to this model produces lower blood pressure, it also reduces fetal pup weights (19). Moreover, existing RAS inhibitors negatively impact fetal health and are thus contraindicated in pregnancy (21).

We have sought to assess the role of the maternal circulating RAS by reduction of maternal hepatic AGT using RNA interference (RNAi). RNAi is a clinically validated technology using synthetic siRNA to specifically and catalytically reduce gene expression via endogenous RNAi machinery (29-31). Conjugation of chemically modified siRNA to triantennary $\mathrm{N}$-acetylgalactosamine provides for hepatocyte-specific uptake via the asialoglycoprotein receptor and stable target suppression $(32,33)$. We have used such siRNAs for evaluating the impact of Agt suppression in models of preeclampsia. As a large molecule with liver-specific targeting, this approach may also avoid the fetotoxicity observed with conventional small-molecule RAS inhibitors.

\section{Results}

To demonstrate the ability of hAgt-targeting siRNA to silence hAGT in our preeclamptic rat model, we investigated human and rat Agt mRNA expression levels in maternal liver, kidney, mesometrial triangle, placenta, fetal liver, fetal kidney, and fetal brain from preeclamptic rats treated with hAgt-targeting siRNA compared with preeclamptic rats treated with luciferase-targeting siRNA. Expression analysis showed efficient suppression of $h A g t$ mRNA expression in maternal liver. Maternal kidney showed no differential $h A g t$ mRNA expression between groups (Figure 1A). We confirmed that silencing of hAgt has no impact on rat AGT $(r A g t)$ mRNA expression in maternal liver or kidney (Figure 1B). Moreover, $h A g t$ mRNA expression in mesometrial triangle as well as placenta, fetal liver, fetal kidney, and fetal brain was not suppressed by $h A g t$-targeting siRNA and suggested that siRNA does not cross the placental barrier (Figure 1C). rAgt expression was also unaffected by siRNA in mesometrial triangle, placenta, fetal kidney, and fetal brain (Figure 1D). By quantifying the antisense stand (the active strand) in tissue, we could confirm that the siRNA does not appear to be substantially retained in the placenta and is not detectable in fetal tissue. siRNA concentrations were below the limit of quantification in fetal liver, while maternal liver contained high levels of siRNA (Figure 1E). On average, maternal liver exposure (as nanograms of antisense strand per gram of tissue) was approximately 266 times greater than that in the placenta, and approximately 31,661 times greater than that observed in the fetus (based on lower limit of quantitation), consistent with targeted maternal hepatic delivery.

Analysis of serum AGT levels confirmed silencing of hAgt. Serum levels of hAGT were clearly diminished in rats treated with $h A g t$-targeting siRNA compared with luciferase-targeting siRNA as control (Figure 2A). Furthermore, circulating rAGT was decreased in hAgt-targeting siRNA-treated animals (Figure 2B). Investigation of circulating angiotensin peptide levels also revealed a significant reduction as compared with controls. Angiotensin metabolites Ang 1-5, Ang 1-7, Ang 1-8, Ang 1-10, Ang 3-7, and Ang 3-8 were decreased in animals treated with $h A g t$-targeting siRNA (Figure 2C).

We then examined the effect of $h A g t$-targeting siRNA on the preeclamptic phenotype, where we found an impact on the preeclampsia-defining symptoms of hypertension and proteinuria. Radiotelemetrically measured mean arterial pressure (MAP) was significantly 
A

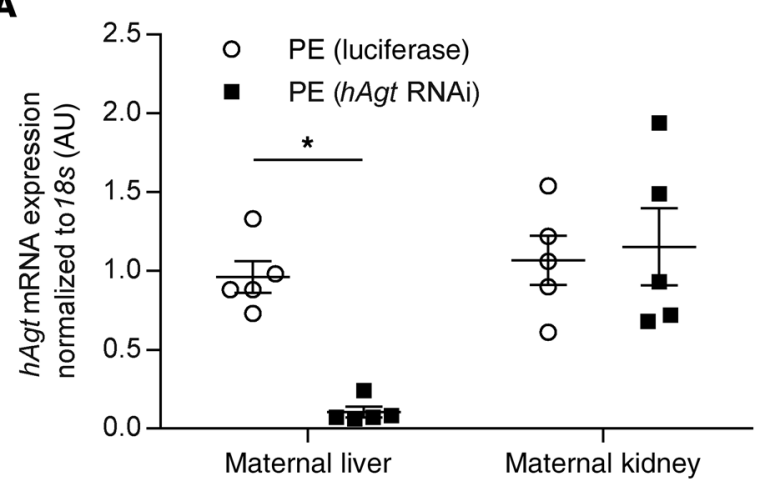

C

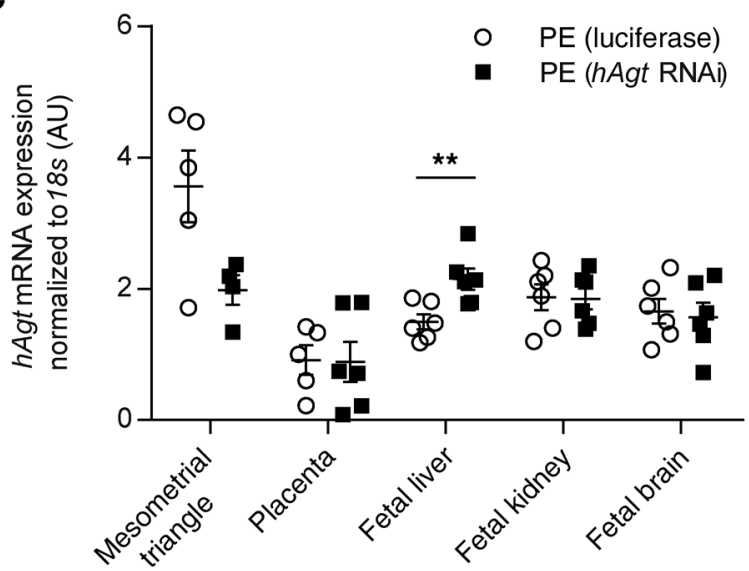

E

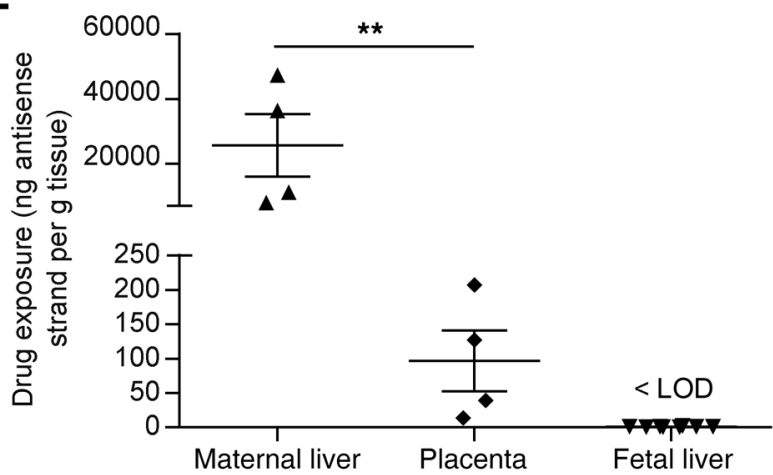

B

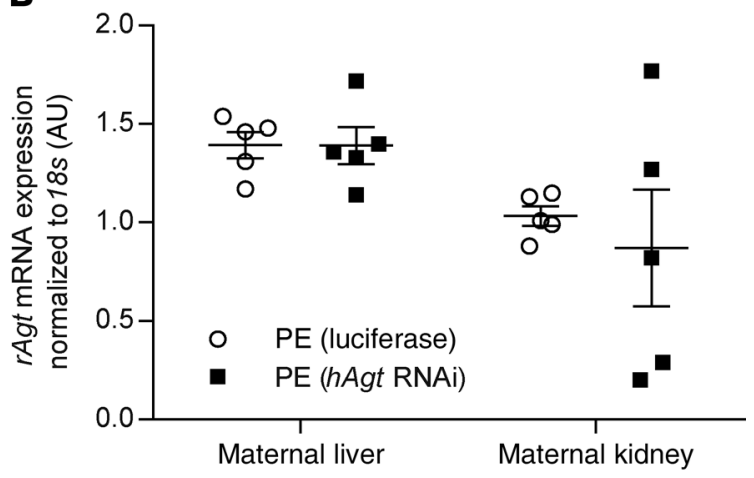

D

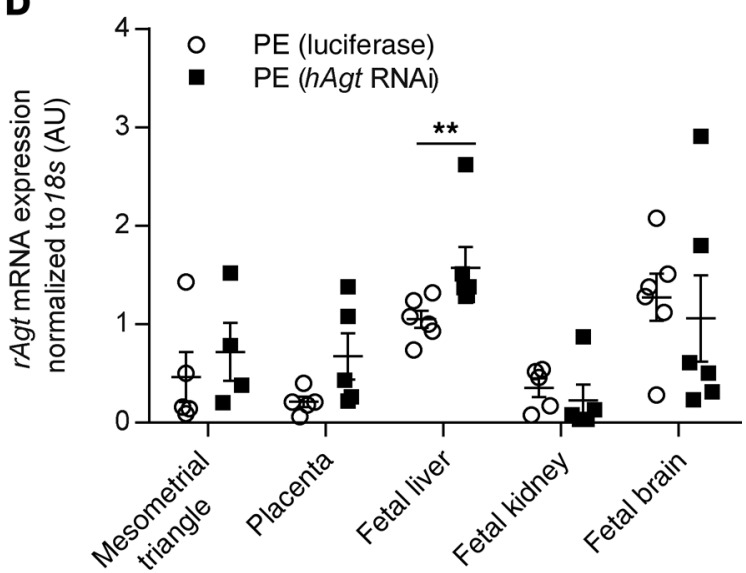

Figure 1. siRNA does not cross the placental barrier. (A) Effect of luciferase-targeting siRNA or hAgt-targeting siRNA on hAgt mRNA expression levels in maternal liver and kidney tissue of preeclamptic (PE) rats. hAgt expression was significantly reduced in maternal liver but not in maternal kidney $(n=5$ each; ${ }^{*} P<0.05$; Mann-Whitney test; mean \pm SEM). (B) Effect of luciferase-targeting siRNA or $h A g t$-targeting siRNA on rAgt mRNA expression levels in maternal liver and kidney tissue of PE rats. rAgt expression was unaffected in maternal liver and kidney by siRNA ( $n=5$ each; mean \pm SEM). (C) Effect of luciferasetargeting siRNA or $h A g t$-targeting siRNA on $h A g t$ mRNA expression levels in mesometrial triangle, placenta, and fetal tissue in PE rats. hAgt expression was not suppressed by $h A g t$-targeting siRNA in mesometrial triangle, placenta, fetal liver, fetal kidney, or fetal brain. Expression level of $h A g t$ was higher in fetal liver of $h$ Agt-targeting versus luciferase-targeting siRNA-treated rats $\left(n=5\right.$ each; ${ }^{*} P<0.05,{ }^{* *} P<0.01$; unpaired $t$ test; mean \pm SEM). (D) Effect of luciferasetargeting siRNA or $h A g t$-targeting siRNA on rAgt mRNA expression levels in mesometrial triangle, placenta, and fetal tissue of PE rats. $r A g t$ expression was unaffected in mesometrial triangle, placenta, fetal kidney, and fetal brain by siRNA. Expression level of $r A g t$ was higher in fetal liver of $h A g t$-targeting versus luciferase-targeting siRNA-treated rats $\left(n=5\right.$ each; ${ }^{*} P<0.01$; Mann-Whitney test; mean \pm SEM). (E) Distribution of $h A g t$-targeting siRNA in PE rats after treatment. The highest amount of siRNA could be detected in the maternal liver $(n=4)$, whereas the amount of siRNA was lower in placental compared with maternal liver $\left(n=4 ;{ }^{* *} P<0.01\right.$; 1-way ANOVA with Tukey's post hoc test; mean \pm SEM) and none was detectable in fetal liver $(n=8)$. LOD, limit of detection.

reduced over the course of pregnancy in rats treated with $h$ Agt-targeting siRNA compared with luciferase-targeting siRNA (Figure $3 A)$. Silencing of $h A G T$ prevented the increase in blood pressure in the last trimester of pregnancy (MAP on day 16 of gestation: 156.24 $\pm 1.89 \mathrm{mmHg}$ luciferase vs. $138.44 \pm 1.81 \mathrm{mmHg} h A g t \mathrm{RNAi}$ ), as well as substantially reduced 24-hour urine albumin secretion (18.04 \pm $3.19 \mathrm{mg} / \mathrm{d}$ luciferase vs. $2.75 \pm 0.56 \mathrm{mg} / \mathrm{d}$ hAgt RNAi) (Figure 3B). Additionally, renal neutrophil gelatinase-associated lipocalin (Ngal) 
A

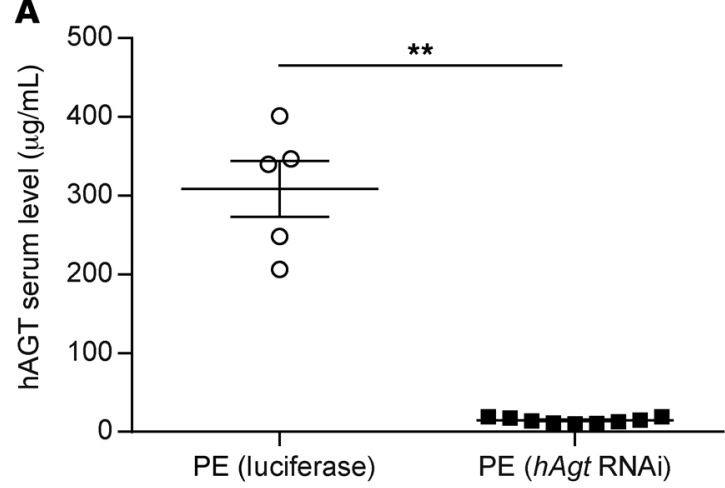

B

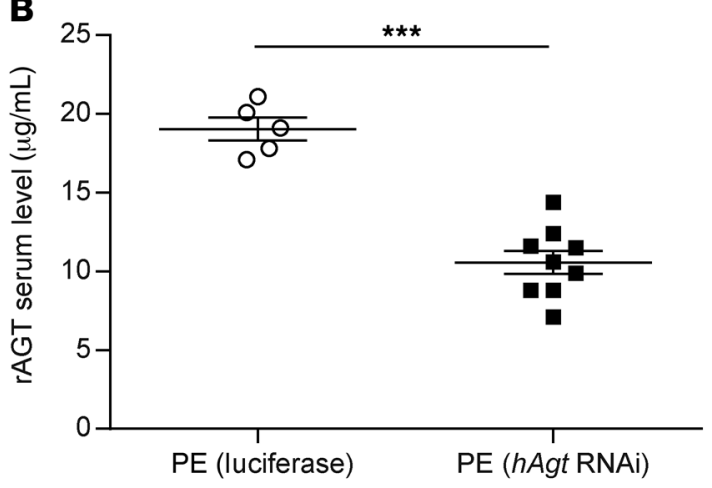

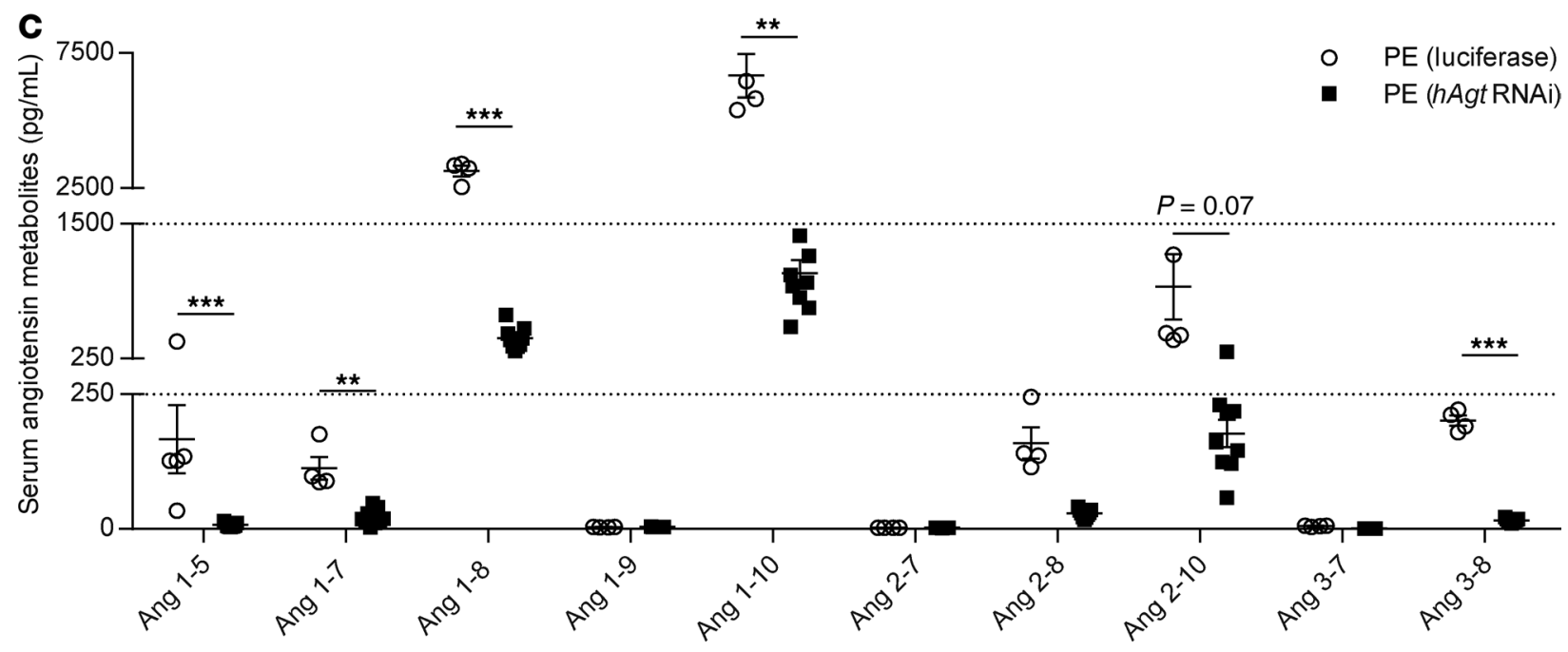

Figure 2. siRNA against $h$ Agt reduces serum angiotensinogen and angiotensin peptide levels. (A) Effect of luciferase-targeting siRNA or $h A g t$-targeting siRNA on serum hAGT levels in preeclamptic (PE) rats. Serum hAGT concentrations were significantly reduced in $h A g t$-targeting siRNA-treated $(n=9)$ versus luciferase-targeting siRNA-treated $(n=5)$ rats ( ${ }^{*} P<0.01$; unpaired $t$ test; mean \pm SEM). (B) Effect of luciferase-targeting siRNA or $h A g t$-targeting siRNA on serum rAGT levels in PE rats. Serum rAGT concentrations were significantly reduced in $h A g t$-targeting siRNA-treated $(n=9)$ versus luciferase-targeting siRNA-treated $(n=5)$ rats ( ${ }^{* *} P<0.001$; unpaired $t$ test; mean \pm SEM). (C) Effect of luciferase-targeting siRNA or $h A g t$-targeting siRNA on circulating angiotensin (Ang) peptide levels in PE rats. Serum peptide concentrations of Ang 1-5, Ang 1-7, Ang 1-8, Ang 1-10, and Ang 3-8 were lower in hAgt-targeting siRNA-treated $(n=9)$ versus luciferase-targeting siRNA-treated $(n=5)$ rats $\left({ }^{* *} P<0.01,{ }^{* * *} P<0.001\right.$; Mann-Whitney test or unpaired $t$ test; mean \pm SEM). Serum peptide concentrations of Ang 1-9, Ang 2-7, Ang 2-8, Ang 2-10, and Ang 3-7 were unaffected.

mRNA expression was significantly decreased $(P<0.05)$ in $h A g t$-targeting siRNA-treated rats (Figure $3 \mathrm{C}$ ). Serum creatinine and serum cystatin $\mathrm{C}$ were within the normal range and were not affected by the treatment (Supplemental Figure 1; supplemental material available online with this article; https://doi.org/10.1172/JCI99417DS1).

Improvement of the maternal phenotype was accompanied by an improvement of the fetal phenotype. Fetal weights on day 21 of gestation were significantly higher in $h A g t$-targeting siRNA-treated rats $(3.40 \pm 0.02 \mathrm{~g})$ compared with luciferase-targeting siRNAtreated animals $(2.64 \pm 0.03 \mathrm{~g})$ (Figure $4 \mathrm{~A})$. Fetal weight frequency distribution curves demonstrated that all fetuses treated with hAgt-targeting siRNA were above the 10th percentile and the numbers of markedly underdeveloped pups were manifestly reduced (Figure 4B). Additionally, analysis of the fetuses of dams treated with $h A G T$-targeting siRNA indicated a positive influence on FGR, as assessed on the 21st day of pregnancy. The FGR ratio is calculated by division of the weight of fetal brains by the weight of fetal livers (Figure 4C). Application of hAgt-targeting siRNA decreased the fetal brain/liver weight ratio in comparison with luciferase-tar- geting siRNA-treated animals $(0.88 \pm 0.02$ luciferase vs. $0.73 \pm 0.02$ $h A g t$ RNAi). Fetal brain and liver weights were higher in $h A g t$-targeting siRNA-treated rats compared with luciferase-targeting siRNAtreated rats (Supplemental Figure 2, A and B). Uteroplacental unit weight $(0.49 \pm 0.01 \mathrm{~g}$ luciferase vs. $0.61 \pm 0.01 \mathrm{~g} h$ Agt RNAi) was also enhanced by $h A g t$-targeting siRNA application (Supplemental Figure 2C). The numbers of implantation sites and live fetuses were similar between the groups, although there was a trend $(P=0.09)$ toward a decrease in number of fetuses that were reabsorbed in $h$ Agt-targeting siRNA-treated rats (Supplemental Figure 3).

We next investigated whether silencing of hAgt had an impact on trophoblast function and uteroplacental morphology. The area of interstitial trophoblast cell invasion into the mesometrial triangle was increased in $h A g t$-targeting siRNA-treated rats compared with luciferase-targeting siRNA-treated rats $\left(4.18 \pm 0.64 \mathrm{~mm}^{2}\right.$ luciferase vs. $5.93 \pm 0.45 \mathrm{~mm}^{2}$ hAgt RNAi) (Figure 5, A and B). Histomorphological analysis revealed no difference in the area of the mesometrial triangle (Figure $5 \mathrm{C}$ ), whereas a significant overall increase of the placental area in $h A g t$-targeting siRNA-treated rats was detected 
A

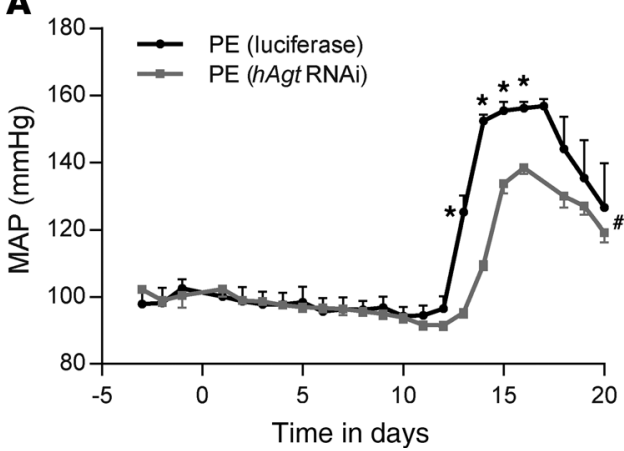

B

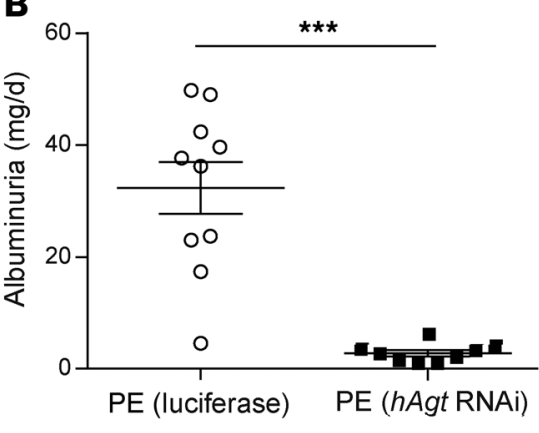

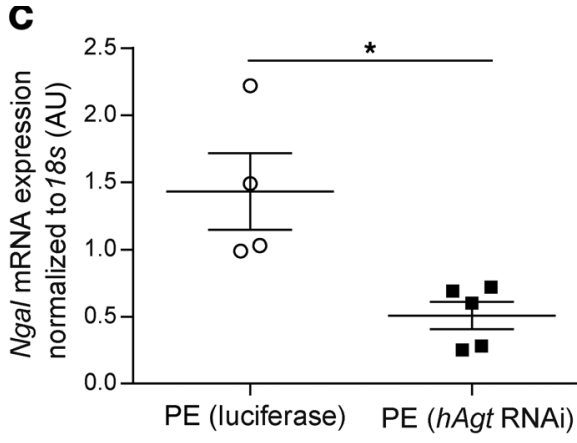

Figure 3. siRNA against $\boldsymbol{h A g t}$ reduces mean arterial pressure and albuminuria. (A) Effect of luciferase-targeting siRNA or $h A g t$-targeting siRNA on mean arterial pressure (MAP) in preeclamptic (PE) rats. MAP measured by telemetry was reduced in hAgt-targeting siRNA-treated ( $n=9$ ) compared with luciferase-targeting siRNA-treated $(n=5)$ PE rats $\left({ }^{*} P<0.01\right.$, ${ }^{\#} P<0.5$; 2-way ANOVA with Bonferroni's post hoc test; mean \pm SEM). (B) Effect of luciferase-targeting siRNA or $h$ Agt-targeting siRNA on albuminuria in PE rats. Albuminuria was normalized by $h$ Agt-targeting siRNA treatment ( $n=9$ ) compared with luciferase-targeting siRNA treatment $(n=10)\left({ }^{* *} P<0.001\right.$; unpaired $t$ test; mean \pm SEM). (C) Effect of luciferase-targeting siRNA or $h A g t$-targeting siRNA on $N g a l$ mRNA expression levels in maternal liver and kidney tissue of PE rats. Ngal expression was significantly reduced in maternal kidney of $h A g t$-targeting versus luciferase-targeting siRNA-treated rats ( $n=5$ each; ${ }^{*} P<0.05$; Mann-Whitney test; mean \pm SEM).

(28.12 $\pm 1.59 \mathrm{~mm}^{2}$ luciferase vs. $33.79 \pm 1.31 \mathrm{~mm}^{2}$ hAgt RNAi) (Figure 5D). This increase was due to the enlargement of the labyrinth zone of the placenta, while the trophospongium area remained unaltered between $h A g t$-targeting siRNA-treated and luciferase-targeting siRNA-treated rats (Figure 5, E and F). Analysis of spiral artery remodeling of the uteroplacental unit revealed an improvement by treatment with $h A g t$-targeting siRNA, suggesting improved placental perfusion (Supplemental Figure 4). Furthermore, we examined the macrophages in the uteroplacental unit. Immunohistological analysis indicated an increase in number of macrophages in the uteroplacental unit in hAgt-targeting siRNA-treated rats compared with luciferase-targeting siRNA-treated rats (Figure 5, G and H).

Since this preeclampsia model features autoantibodies directed against angiotensin II receptor type 1 (AT1-AA), we next investigated whether silencing of $h A g t$ could influence AT1-AA levels (15, 26). Application of $h A g t$-targeting siRNA reduced the presence of AT1-AA (Figure 6). Furthermore, we examined whether silencing of $h A g t$ had an impact on the balance between proangiogenic and antiangiogenic factors, such as placental growth factor (PLGF) and soluble fms-like tyrosine kinase-1 (sFLT-1), since both factors are altered in preeclampsia (34). As shown in Figure 7A, serum PLGF concentration was increased in $h A g t$-targeting siRNA-treated rats compared with luciferase-targeting siRNA-treated rats, whereas serum sFLT-1 (Figure 7B) was reduced. Consequently, the serum sFLT-1/PLGF ratio was decreased (Figure 7C) with $h A g t$ suppression in the transgenic preeclamptic rat model.

Next, we examined the effect of silencing maternal rAgt on blood pressure, uteroplacental unit, and fetal growth in the RUPP model of preeclampsia in rats. Investigation of $r A g t$ mRNA expression levels in maternal liver showed suppression of $r A g t$ mRNA, indicating efficient gene silencing in maternal liver (Figure 8A) in treated rats. Maternal kidney showed no difference in $r A g t$ mRNA expression between groups (Figure 8B). Moreover, rAgt mRNA expression in mesometrial triangle as well as placenta, fetal liver, fetal kidney, and fetal brain was not suppressed by $r$ Agt-targeting siRNA in treated rats (Figure $8, \mathrm{C}-\mathrm{G}$ ).
A

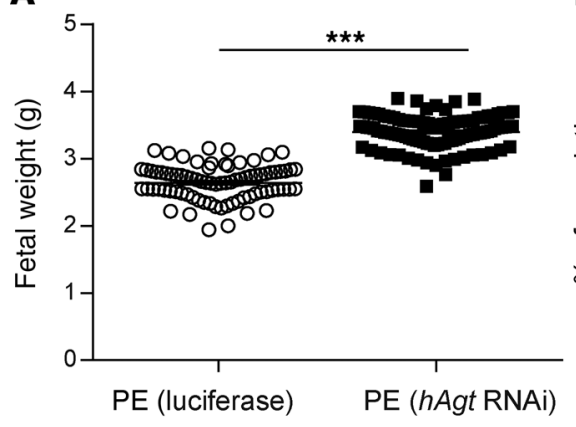

B

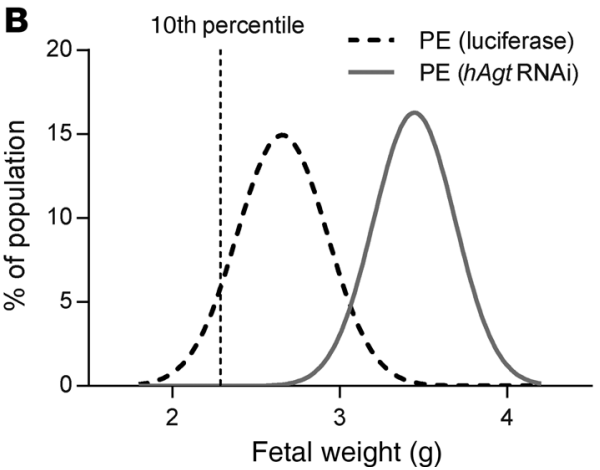

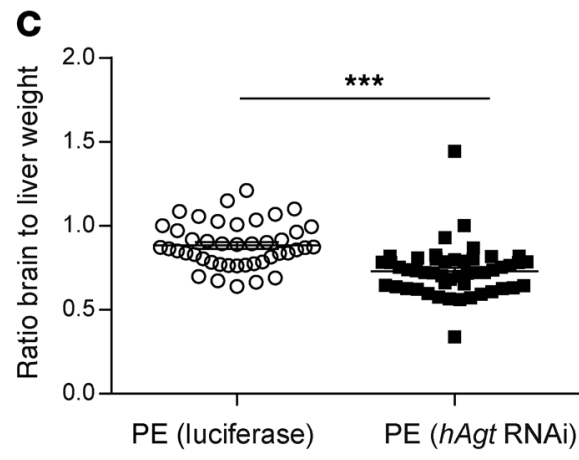

Figure 4. siRNA against $\boldsymbol{h A g t}$ improves fetal outcome. (A) Fetal weights were increased after application of $h$ Agt-targeting siRNA ( $n=102$ ) versus luciferase-targeting siRNA $(n=80)\left({ }^{* *} P<0.001\right.$; unpaired $t$ test; mean \pm SEM). (B) Frequency distribution curves for individual pups. A rightward shift of the curve for pups from the $h A G T$-targeting siRNA group (gray solid curve) compared with the luciferase-targeting siRNA group (black dashed curve) illustrates the improved fetal outcome. Vertical dashed line denotes the 10th percentile of luciferase-targeting siRNA-treated PE fetal weights. (C) Fetal growth restriction is shown as the ratio between fetal brain and liver weights. Application of $h$ Agt-targeting siRNA $(n=49)$ decreased the fetal brain/liver weight ratio compared with luciferase-targeting siRNA $(n=44)\left({ }^{* *} P<0.001\right.$; Mann-Whitney test; mean \pm SEM). 
A

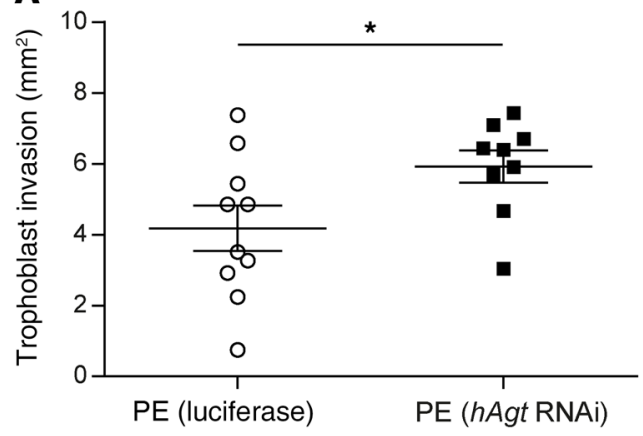

C

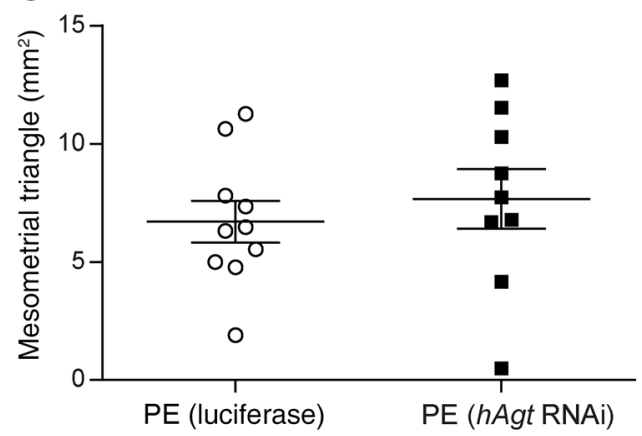

E

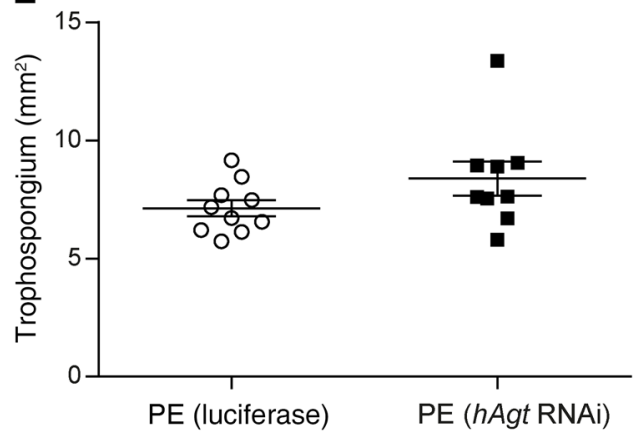

B

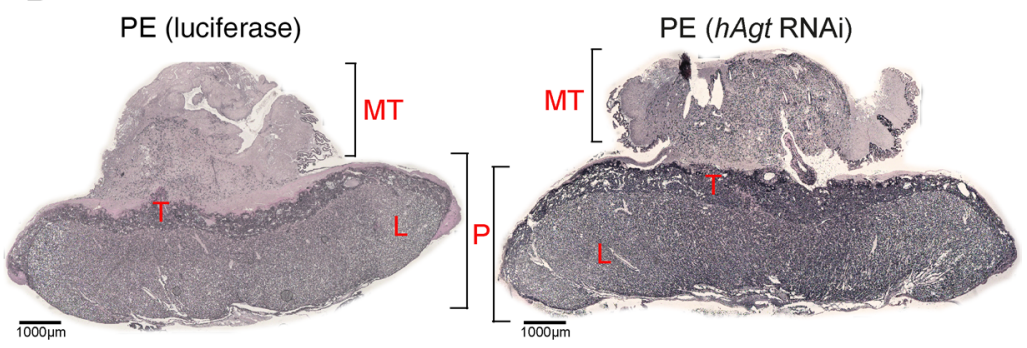

D

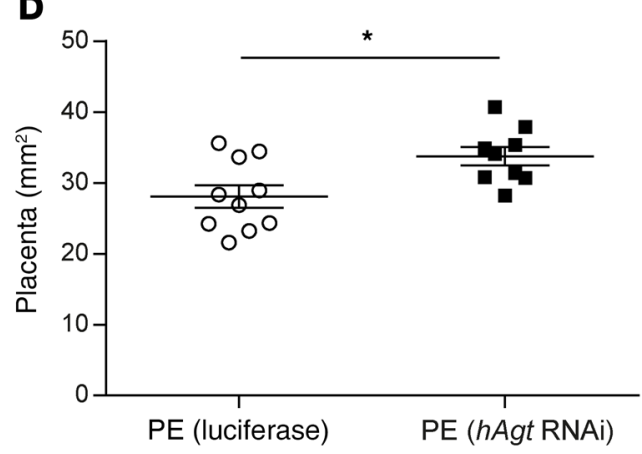

$\mathbf{F}$

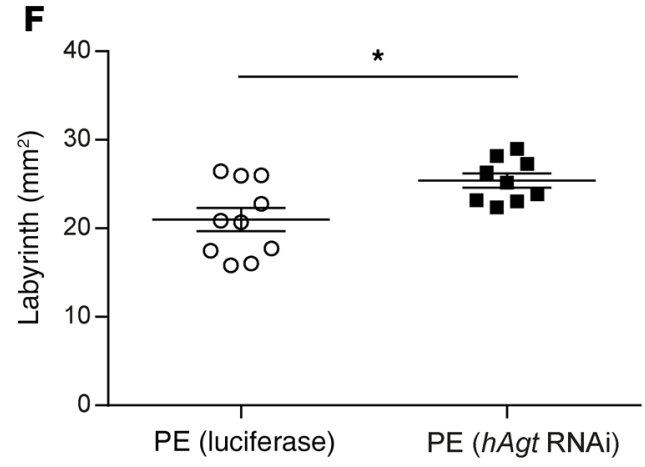

a

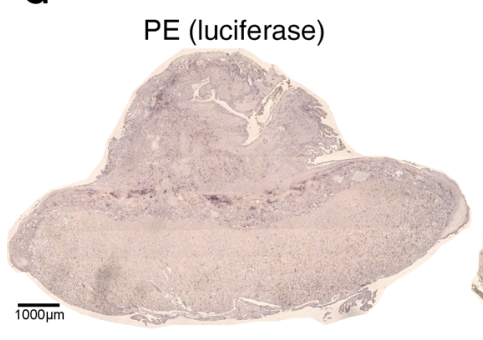

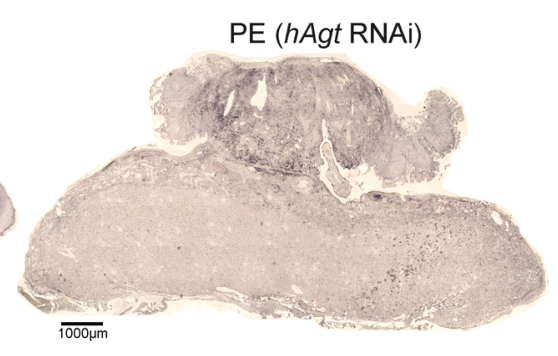

H

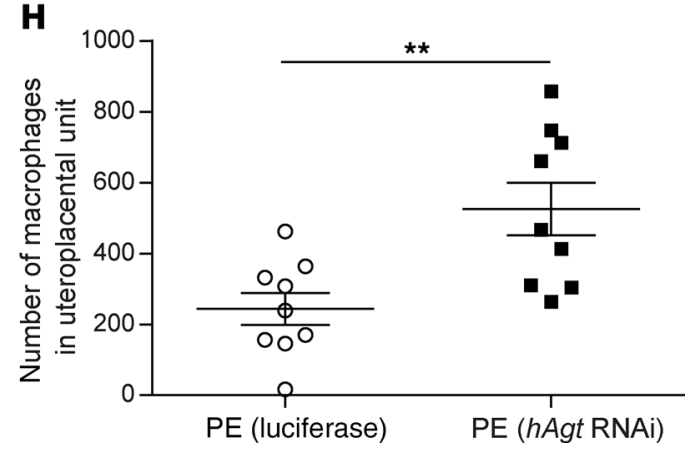

Figure 5. siRNA against hAgt improves placenta morphology. (A) Extension of trophoblast cell invasion was measured as the area of the mesometrial triangle invaded by trophoblast cells. This area was significantly increased in hAgt-targeting siRNA-treated $(n=9)$ preeclamptic (PE) rats compared with luciferase-targeting siRNA-treated $(n=10)$ rats $\left({ }^{*} P<0.05\right.$; unpaired $t$ test; mean \pm SEM). (B) Representative pictures of cytokeratin-stained midsagittal mesometrial triangle (MT) and placental (P) tissue sections used to carry out the histomorphological analysis. Different zones of the placenta are marked: T, trophospongium; L, labyrinth. (C) The area of the mesometrial triangle was not different between luciferase-targeting siRNA- and $h A C T$-targeting siRNA-treated PE rats (mean \pm SEM). (D) The overall size of the placenta was increased in $h A g t$-targeting siRNA-treated $(n=9)$ PE rats compared with luciferase-targeting siRNA-treated $(n=10)$ rats $\left({ }^{*} P<0.05\right.$; unpaired $t$ test; mean \pm SEM). (E) The area of the trophospongium was not changed in hAgt-targeting siRNA-treated $(n=9)$ PE rats compared with luciferase-targeting siRNA-treated $(n=$ $10)$ rats (mean \pm SEM). (F) The area of the labyrinth zone of placentas of $h A g t$-targeting siRNA-treated $(n=9)$ PE rats was enhanced in comparison with luciferase-targeting siRNA-treated $(n=10)$ rats $\left({ }^{*} P<0.05\right.$; unpaired $t$ test; mean \pm SEM). (C) Representative pictures of CD68-stained uteroplacental unit tissue sections. (H) The number of macrophages was increased in hAgt-targeting siRNA-treated $(n=9)$ PE rats compared with luciferase-targeting siRNA-treated $(n=9)$ rats. ${ }^{* *} P<0.01$; unpaired $t$ test. 


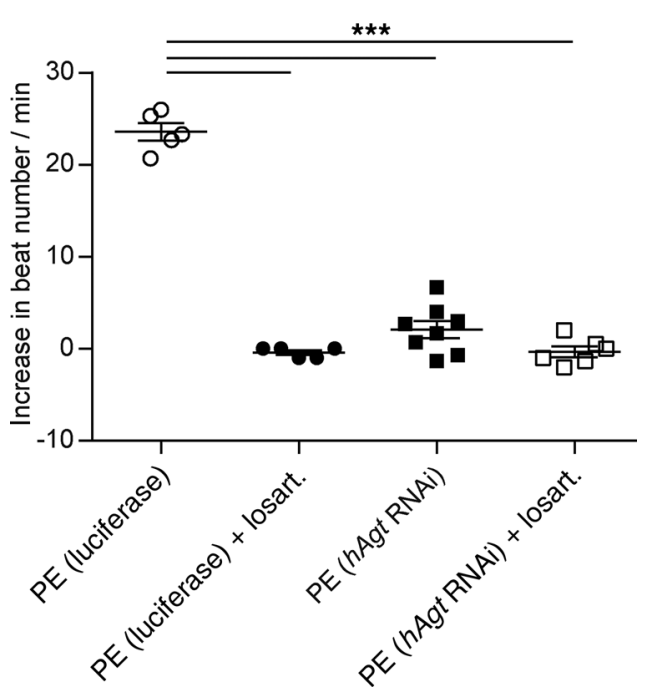

Figure 6. siRNA against hAgt decreases the concentration of angiotensin II receptor type 1 autoantibodies (AT1-AA) in the serum of preeclamptic rats. Isolated rat neonatal cardiomyocytes were treated in vitro with IgG from luciferase-targeting siRNA-treated $(n=5)$ or hAgt-targeting siRNA-treated $(n=8)$ preeclamptic $(P E)$ rats. Data are presented as an increase in beating rate of cardiomyocytes after treatment with IgC compared with baseline. Administration of AT1 receptor blocker (losartan) ( $n=5$ each) was used to show that the effect is mediated via the AT1 receptor ${ }^{* * *} P<0.001 ; 1$-way ANOVA with Tukey's post hoc test; mean \pm SEM).

MAP was elevated in RUPP versus normal pregnant (NP) rats $(135.3 \pm 5.3$ vs. $101.4 \pm 4.5 \mathrm{mmHg})$. Treatment of RUPP rats with $r$ Agt-targeting siRNA led to a reduction in MAP (117.0 \pm $4.9 \mathrm{mmHg}$ ) in comparison with untreated RUPP rats, but there was no significant change in NP rats treated with $r A g t$-targeting siRNA ( $r$ Agt RNAi; $92.1 \pm 2.9 \mathrm{mmHg}$ ) compared with untreated NP rats (Figure 9A). As has been previously described in the RUPP model, we could not detect albuminuria (Figure 9B). Average fetal weights by group were $2.30 \pm 0.09 \mathrm{~g}(\mathrm{NP}), 1.89 \pm 0.10 \mathrm{~g}$ (RUPP), $2.38 \pm 0.09 \mathrm{~g}(\mathrm{NP}[r A g t \mathrm{RNAi}])$, and $2.12 \pm 0.06$ (RUPP [ $r A g t$ RNAi]) (Figure 9C). A 1-way ANOVA with Tukey's post hoc test indicated a significant reduction in pup weights in RUPP compared with NP, but not NP ( $r A g t$ RNAi) or RUPP ( $r A g t$ RNAi), indicating that administration of siRNA to RUPP rats ameliorated the decrease in fetal weight. Although the effect of siRNA treatment on fetal weights in the RUPP group did not reach significance in comparison with untreated RUPP, the weights were also no longer significantly different from NP. Furthermore, the fetal brain/liver weight ratio as a measure of FGR was increased by the RUPP procedure, but reduced with the addition of the siRNA. Administration of rAgt-targeting siRNA to RUPP rats thus improves the FGR (Figure 9D). However, uteroplacental weights were not affected by $r A g t$-targeting siRNA treatment in the RUPP model (Supplemental Figure 5A). The numbers of implantation sites, live fetuses, and fetuses that were reabsorbed were similar between untreated and siRNA-treated NP and RUPP rats (Supplemental Figure 5, B-D).

As with the transgenic model, the RUPP procedure induces the production of activating autoantibodies against the AT1. Similar to the results in the transgenic rat model, silencing of $r A g t$ leads to a normalization of activity (Supplemental Figure 6). Neither the
RUPP procedure nor suppression of $r A g t$ by siRNA had an impact on the balance between proangiogenic (PLGF) and antiangiogenic (sFLT-1) factors (Supplemental Figure 7).

Next, we looked in more detail into whether the rAgt siRNA treatment has any negative consequences for the fetus. We treated NP rats with $r A g t$ siRNA, using the same regimen as with the RUPP model, for comparison with untreated NP controls. We performed micro-CT ( $\mu \mathrm{CT})$ of the skull of the fetus at the end of the pregnancy to analyze calvarial morphology. We could not detect any changes in the fetal skull, as both width and length were unchanged (Figure $\left.10, \mathrm{~A}^{-} \mathrm{C}\right)$. Also, the weight and volume of fetal brain at the end of gestation and in 3-week-old offspring showed no effect of $r A g t$ siRNA (Supplemental Figure 8, A-D). Moreover, we analyzed the expression of different genes that are important during brain development, such as adenosine receptor 1 (Adora, neuromodulation), synaptophysin (Syp1, synaptic plasticity), neuregulin-1 (Nrg1, neurogenesis), and neuropilin-1 (Nrp1, axonal growth) $(35,36)$, and could not detect an influence of siRNA treatment in the fetal brain (Supplemental Figure 8, E-H). Next, we examined the impact of the $r A g t$ siRNA treatment on renal development in the fetus. We could not detect any difference in kidney weight/body weight ratio in 3-weekold offspring from dams that were treated with siRNA (Figure 10D). The histological analyses of offspring kidney revealed no alteration in renal morphology by the siRNA treatment (Figure 10, E-G). Additionally, we analyzed the expression of different genes that are important during kidney development, including octamer-binding transcription factor 4 (Oct4, renal stem/progenitor cells), paired box gene 2 (Pax2, intermediate mesoderm), sine oculis-related homeobox 2 (Six2, nephron progenitor cells), and homeobox protein B7 (HoxB7, ureteric bud) $(37,38)$, and could not detect an influence of siRNA treatment in fetal kidney at the end of pregnancy or in 3-week-old offspring (Supplemental Figure 9). We looked next at the effect of the siRNA treatment on the fetal heart. We could not detect any difference in heart weight/body weight ratio in fetuses and offspring from dams that were treated with siRNA (Supplemental Figure 10, A and B). To investigate whether a persistent patent ductus arteriosus occurs in the fetus and offspring, we determined circulating prostaglandin $\mathrm{E}_{2}\left(\mathrm{PGE}_{2}\right)$ levels. It has been shown that prostaglandins maintain the patency of the ductus arteriosus in the fetus, and some studies have found that preterm infants with patent ductus arteriosus have significantly higher levels of $\mathrm{PGE}_{2}$ (39-41).

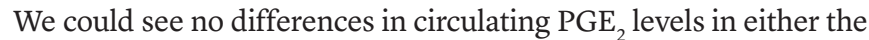
fetus or the offspring with siRNA treatment (Supplemental Figure 10, C and D). Finally, we studied offspring lungs to see whether there was pulmonary hyperplasia due to siRNA treatment. We could not detect any difference in lung weight/body weight ratio in 3-week-old offspring from dams that were treated with siRNA (Supplemental Figure 10E). As a morphometric index, we determined the mean linear intercept $(\mathrm{Lm})$ as an index of alveolar size. The mean linear intercept in offspring lung was not affected by siRNA treatment during pregnancy (Supplemental Figure 10, E-H).

\section{Discussion}

In the present study we have demonstrated that RNAi therapeutics targeting Agt ameliorated the preeclamptic phenotype in 2 animal models of preeclampsia. These models reflect different approaches to producing the preeclamptic phenotype in rodents. With this 

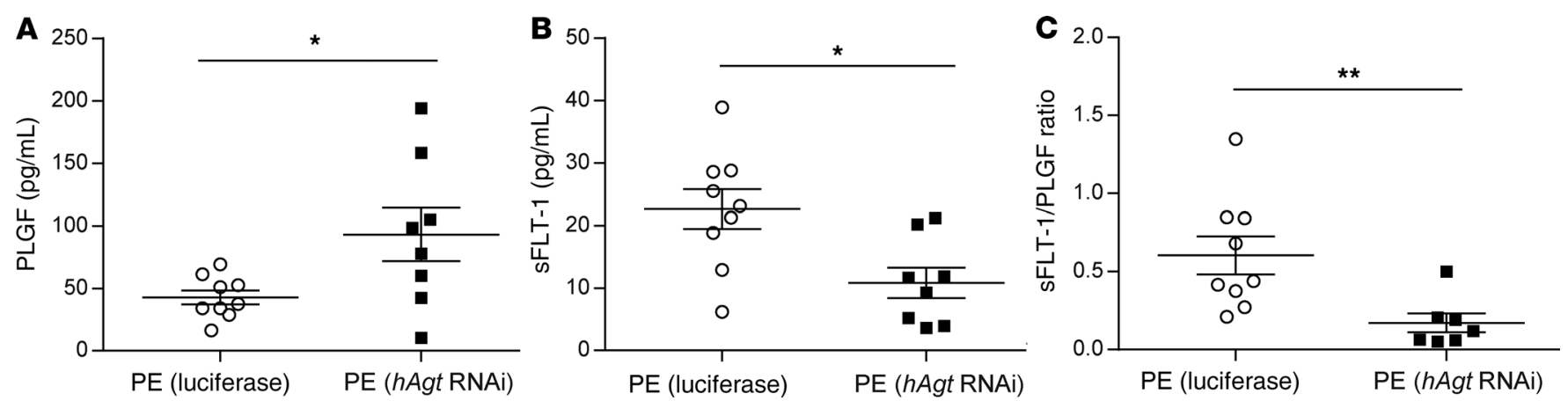

Figure 7. siRNA against $\boldsymbol{h A g t}$ alters proangiogenic and antiangiogenic factors. (A) Serum PLGF concentrations were increased in $h A g t$-targeting siRNA-treated $(n=8)$ versus luciferase-targeting siRNA-treated $(n=10)$ rats $\left({ }^{*} P<0.05\right.$; unpaired $t$ test; mean \pm SEM). (B) Serum sFLT-1 concentrations were reduced in $h A g t$-targeting siRNA-treated $(n=8)$ versus luciferase-targeting siRNA-treated $(n=10)$ rats $\left({ }^{*} P<0.05\right.$; unpaired $t$ test; mean \pm SEM). (C) The sFLT-1/PLGF ratio was decreased in $h$ Agt-targeting siRNA-treated $(n=8)$ versus luciferase-targeting siRNA-treated $(n=10)$ rats $\left({ }^{* *} P<0.01\right.$; unpaired $t$ test; mean \pm SEM).

technique, we were able to selectively modulate maternal RAS signaling, while preserving the fetal RAS in both models. Similar levels of Agt suppression by siRNA have been described previously, in nongravid spontaneously hypertensive rats (42). In that study, approximately $98 \%$ suppression of $A g t$ by RNAi was well tolerated and produced substantial blood pressure lowering. Thus, while means of reversing siRNA pharmacology exist (43), as presently evaluated, they do not appear necessary. However, further studies are necessary to assess whether conventional means of raising blood pressure remain effective with Agt suppression, particularly in the context of pregnancy.

In the transgenic model, silencing of hAgt only in the maternal liver decreased the elevated AGT and angiotensin metabolite levels in the tissue and circulating renin-angiotensin systems, leading to a reduction of blood pressure and urinary albumin excretion. We improved FGR, reversing the brain-sparing phenomenon and demonstrating increased brain size, indicating an improved fetal development. Upregulation of $h A g t$ and $r A g t$ was noted in the fetus. The cause and the consequences of Agt upregulation in the fetal liver are unclear and deserve further investigation; however, this was not observed in the RUPP model and, in view of improvements in the fetus, may not be deleterious.

The RUPP model confirmed the principal findings of the first model, including improved FGR and increased fetal brain size, in a model that is not explicitly driven by the RAS. Critically, fetal weights were not reduced in the treated group, and blood pressure was lowered. Moreover, a detailed assessment of brain, lung, kidney, and heart in fetus and offspring of rAgt-treated dams revealed no deleterious effects. In particular, siRNA treatment did not lead to renal tubular dysgenesis, calvarial or pulmonary hypoplasia, or persistent patent ductus arteriosus. In summary, we can say that in all studies regarding the impact of siRNA treatment on the fetus, we did not detect any negative fetal consequences of the treatment. Thus, through use of a maternal-targeted agent, we believe we have uncoupled the preeclamptic treatment conundrum: that blood pressure reduction is associated with a reduction in fetal weight.

Our studies give further insight into the potential benefits of blocking the maternal RAS. The first model acts by upregulation of the circulating and uteroplacental RAS (15). The dams demonstrate placental insufficiency with severe FGR, resembling early-onset preeclampsia. In the first model, silencing of $h A g t$ enhances the area of interstitial trophoblast cell invasion, as well as the placenta area. AT1 autoantibodies, reported to play a pathological role in preeclampsia $(44,45)$, were significantly reduced. The sFLT-1/PLGF ratio, considered not only to play a role in pathophysiology but also to be a potential biomarker of disease progression, was improved as well (46).

The improvement of both the maternal and fetoplacental phenotypes in the transgenic rat model confirms the role of the circulating RAS in the progression of the disease in this model, and indicates the significant impact circulating factors can have on the fetus and placenta. Further studies are necessary to determine whether reduction of maternal AGT levels leads to a reduction of oxidative stress or other known features of the human condition not presently evaluated (5). How maternal AGT and its active metabolites affect placental development is a key question.

Although blood pressure was not fully normalized in the present model, neither were angiotensin metabolites fully normalized to levels observed in normotensive pregnant rats. Treatment reduced angiotensin II by $90 \%$ in comparison with control transgenic animals, but angiotensin II remained elevated over that in normotensive pregnant controls. Considering AGT, treatment reduced hAGT to $5 \%$ of that of control animals; rAGT, though not targeted by the siRNA, was also reduced to nearly half that of control transgenic animals. Absent any change in transcription, this suggests an increase in conversion of rAGT to angiotensin I. Given that angiotensin II is known to inhibit renin secretion (47), near-elimination of angiotensin II derived from the human isoform may have resulted in a derepression of rat renin and subsequently enhanced angiotensin production from the rat AGT isoform.

Although no animal model of preeclampsia fully recapitulates the clinical phenotype, one important aspect of our results and the transgenic rat model in particular is the clear translation of the intervention in rats to the human situation. In patients, we aim for a modest reduction in blood pressure (to a systolic blood pressure of $\sim 140-160 \mathrm{mmHg}$ ) without compromising effects on the fetus, as excessive blood pressure lowering is associated with FGR (23). Here, we have similarly reduced blood pressure and indeed enhanced fetal outcomes (such as 

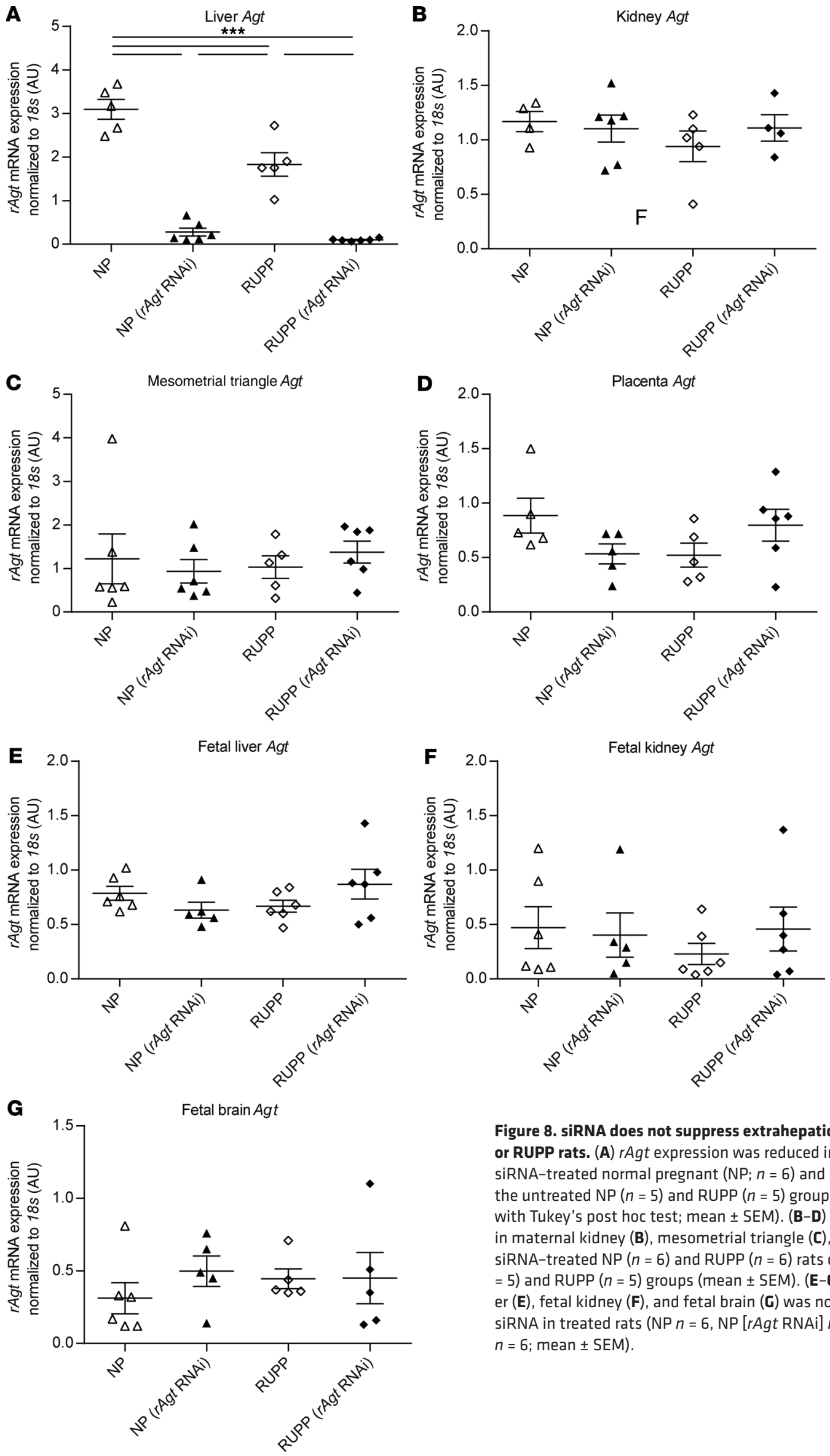

Figure 8. siRNA does not suppress extrahepatic or fetal Agt in normal pregnant or RUPP rats. (A) rAgt expression was reduced in maternal liver in $r$ Agt-targeting siRNA-treated normal pregnant (NP; $n=6)$ and RUPP $(n=6)$ rats compared with the untreated NP $(n=5)$ and RUPP $(n=5)$ groups $\left({ }^{* *} P<0.001\right.$; 1-way ANOVA with Tukey's post hoc test; mean \pm SEM). (B-D) rAgt expression was not altered in maternal kidney (B), mesometrial triangle (C), or placenta (D) in rAgt-targeting siRNA-treated NP $(n=6)$ and RUPP $(n=6)$ rats compared with the untreated NP ( $n$ $=5$ ) and RUPP $(n=5)$ groups (mean \pm SEM). (E-C) rAgt mRNA expression in fetal liver (E), fetal kidney (F), and fetal brain (C) was not downregulated by rAgt-targeting siRNA in treated rats (NP $n=6$, NP [rAgt RNAi] $n=5$, RUPP $n=6$, RUPP [rAgt RNAi] $n=6$; mean \pm SEM) 

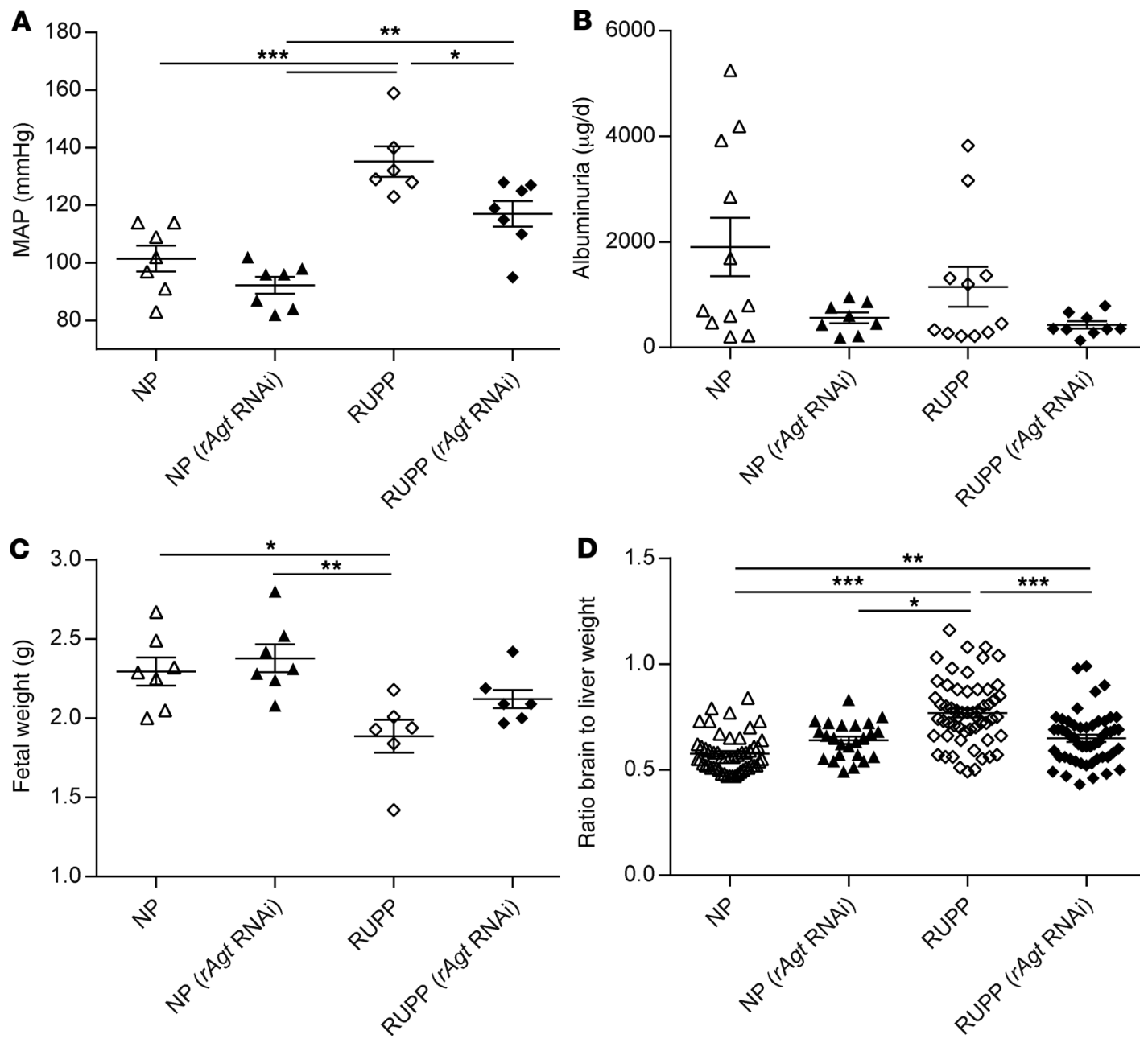

Figure 9. Administration of rAgt siRNA to RUPP rats blunts the increase in blood pressure and prevents a decrease in fetal weights. (A) MAP was reduced in rAgt-targeting siRNA-treated RUPP rats $(n=7)$ compared with untreated RUPP rats $(n=6)$, and unchanged in NP rats treated with $r A g t$ targeting siRNA $(n=7)$ compared with untreated NP rats $(n=7)\left({ }^{*} P<0.05\right.$, ${ }^{* *} P<0.01$, ${ }^{* * *} P<0.001$; 1-way ANOVA with Tukey's post hoc test; mean \pm SEM). (B) Albuminuria was not observed in the RUPP model, and no differences could be detected between rAgt-targeting siRNA-treated rats and untreated rats (NP $n=11$, NP [rAgt RNAi] $n=8$, RUPP $n=11$, RUPP [rAgt RNAi] $n=9$; mean \pm SEM). (C) Fetal weights were decreased in response to placental ischemia in untreated RUPP rats $(n=6)$ compared with untreated NP rats $(n=7)$ and NP rats treated with $r$ Agt-targeting siRNA ( $n=7)$; however, the application of $r$ Agt-targeting siRNA to RUPP rats $(n=7)$ ameliorated the decrease in fetal weight $\left({ }^{*} P<0.05,{ }^{*} P<0.01 ; 1\right.$-way ANOVA with Tukey's post hoc test; mean \pm SEM). (D) Fetal brain/liver weight ratios were increased in untreated RUPP $(n=6)$ compared with untreated $(n=7)$ and treated $r A g t-$ targeting siRNA-treated $(n=7)$ NP rats. Administration of $r$ Agt-targeting siRNA to RUPP rats $(n=7)$ reduced the fetal brain/liver weight ratio $\left({ }^{*} P<0.05\right.$, ${ }^{* *} P<0.01,{ }^{* *} P<0.001 ; 1$-way ANOVA with Tukey's post hoc test; mean \pm SEM).

reduced FGR). Moreover, we have confirmed the principal findings in an orthogonal model of preeclampsia, the RUPP model. In the present study, complete normalization of blood pressure in the transgenic model may not have been achieved owing to inadequate Agt suppression (including over time), or perhaps to a rise in rat renin (as suggested by reduced full-length rAGT in circulation), offsetting the impact of $h A g t$ lowering. Further studies are warranted to look at fetal outcomes if blood pressure is fully normalized with Agt RNAi.

The second model is a surgical model that induces ischemia/ reperfusion injury and subsequent local and systemic inflammation restriction (28). This model is performed after placentation has taken place, and FGR, while present, is markedly less than in the first model. Thus, the second model represents more a model of late-onset preeclampsia. In the RUPP model, only a single dose of siRNA was given, in the middle of gestation, to avoid potential complications with the RUPP procedure (such as hypotension). Given the expected delay between siRNA dosing and onset of action (9), blood pressure reduction may not have been maximal at time of assay. Thus, optimizing study parameters further could yield further improvements in outcomes.

Despite exposure of the dams to the siRNA at relatively high doses, siRNA in fetal liver was below the limit of detection. These data are consistent with available data on placental transfer indicating limited transfer for large negatively charged molecules without active transport (48). Moreover, the $\mathrm{GalNAc}_{3}$-conjugated siRNA requires receptor-mediated uptake by the asialoglycoprotein receptor, which is not expressed appreciably in fetal liver until near delivery $(49,50)$. Thus, the use of a maternal livertargeted isoform-specific siRNA in a transgenic model allows the 

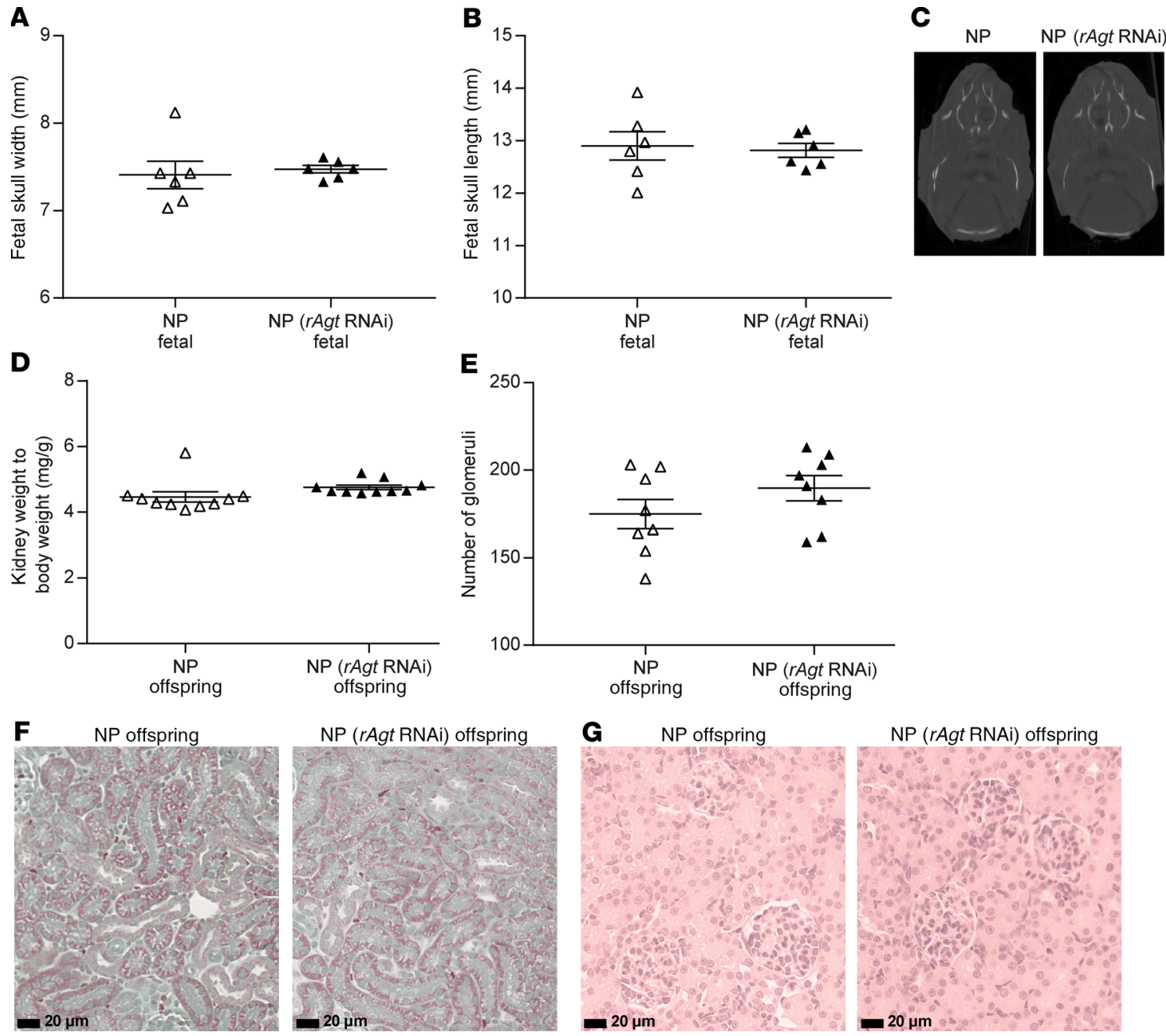

Figure 10. rAgt siRNA has no negative impact on calvarial and renal morphology of the fetus. (A) Measurement of fetal skull width showed no difference in pups from untreated and siRNA-treated NP rats $(n=6$ each; mean \pm SEM). (B) Fetal skull length was also unchanged in pups from untreated and siRNAtreated NP rats ( $n=6$ each; mean \pm SEM). (C) Representative micro-CT images of fetal rat skulls. (D) The kidney weight/body weight ratio of 3-week-old offspring was not changed between untreated and siRNA-treated groups ( $n=10$ each; mean \pm SEM). (E) Number of glomeruli in 3-week-old offspring kidneys was not influenced by siRNA treatment $(n=10$ each; mean \pm SEM). (F) Representative images of Masson's trichome-stained kidney sections of offspring from untreated and siRNA-treated NP rats. (G) Representative images of H\&E-stained kidney sections of offspring from untreated and siRNA-treated NP rats.

separation of primary and secondary effects of the treatment. hAGT expression in the placenta was unchanged, with a trend toward upregulation of rAGT. Upregulation of both isoforms in fetal liver, while an unexpected result, may reflect a response to altered hemodynamics. Consistent with this upregulation, we observed an increase in trophoblast invasion, a process known to be stimulated by local angiotensin II (26). Macrophage presence was also increased. The role of macrophages in preeclampsia is complex, and the physiological impact depends on the class of macrophages present (51). M2 macrophages are thought to mediate tolerance as well as trophoblast invasion (52), both processes relevant to preeclampsia. Although the identity of the macrophages induced by treatment in the present study was not assessed, these data warrant further evaluation of angiotensinmediated alterations in the macrophage population in preeclampsia. These findings may provide a mechanism for the observed larger placenta size in treated animals as well as reduced FGR.
The interplay between AT1-AA and endogenous angiotensin II is unclear, as in vivo studies evaluating the impact of AT1-AA infusion have generally been in the context of normal endogenous angiotensin II levels $(15,44,45)$. Our observation of reduced AT1-AA suggests that angiotensin-mediated signaling impacts the production of these autoantibodies. It has been demonstrated that antibodies generated against the known epitope required infusion of angiotensin II to recapitulate the disease model (53). Additionally, administration of an ACE inhibitor in the infusion model reduced blood pressure by a greater amount in infused animals than in NP animals, indicating that the endogenous ligand remains active and may act synergistically with AT1-AA (19). How the continued activity of angiotensin II relates to the production and activity of AT1-AA requires further elucidation.

Targeting the RAS in a maternal-specific manner may be an effective treatment for preeclampsia, as demonstrated in the present models. The ability to lower maternal blood pressure while 
supporting fetal growth contrasts with existing agents. Drugs such as methyldopa, labetolol, and nifedipine neither address the pathophysiology of preeclampsia, nor support fetal growth. As a result, their use presents a dilemma for physicians who need to balance the health of the mother and the needs of the growing fetus (23). One important aspect of our results is the relevance to the human situation. In patients we aim for a modest reduction in blood pressure (to $140-160 \mathrm{mmHg}$ systolic blood pressure) without compromising effects on the fetus. Further studies are warranted, however, to assess fetal outcomes if blood pressure is normalized. In contrast to antihypertensives currently used in pregnancy, studies have indicated a role for AGT and the RAS in preeclampsia pathophysiology. Particularly relevant is the finding by Ward et al. linking polymorphism associated with higher angiotensin II levels with risk for preeclampsia (16). Other research has demonstrated elevated AT1 levels and potentiated AT1-mediated signaling $(12-14,54)$. Angiotensin II-mediated signaling has been described to stimulate many pathways implicated in preeclampsia (55). For example, application of agonistic AT1 autoantibodies has been observed to result in increased levels of NADPH oxidase in vascular smooth muscle cells (VSMCs) and trophoblasts (56), elevated sFLT-1 in pregnant mice (45), increased tissue factor activity in VSMCs (57), elevated endothelin in pregnant rats (58), and promotion of inflammation in pregnant mice by stimulation of TNF- $\alpha$ (59). Notably, AT1-AA can induce angiotensin sensitivity (53), possibly through impaired internalization of AT1 (60). It also appears that endogenous angiotensin plays a role; for example, enalapril has been observed to blunt the hypertensive effect of AT1-AA in rats (58), while losartan blocks elevation of sFLT-1 in the RUPP model (61). More generally, the associated signaling pathways downstream of AT1 activation have also been defined (62). Given the lack of fetal exposure to the siRNA in the present study, these findings support the further investigation of RNAi therapeutics as a potential treatment for preeclampsia.

Investigational RNAi therapeutics are a clinically validated class of molecules using both targeted delivery and gene-specific silencing. Multiple clinical studies are currently under way across a variety of diseases, including hereditary ATTR amyloidosis, hypercholesterolemia, and hemophilia (63-66). Such clinical programs share a common targeted delivery to the liver. Delivery of RNAi therapeutics via the asialoglycoprotein receptor is highly hepatocyte-specific (32). AGT is predominantly derived from hepatocytes $(9,10)$ and, as the progenitor of the RAS, has widespread effects on physiology (67). As preeclampsia manifests with both maternal and fetal pathology, a therapeutic approach that mitigates the well-described risk of hypertension, reduces the RAS-mediated pathology, and does not impede the fetal or uteroplacental RAS may be beneficial to patients.

We have demonstrated the pivotal role maternal hepatic AGT plays in the progression of preeclampsia in 2 rodent models. Given the differences in disease pathophysiology as well as placentation between rodents and the human condition, it is unclear how directly translatable these findings are. However, supported by the apparent lack of placental transfer, future studies may seek to extend these findings. Critically, further studies are necessary to determine whether such a correction of the disease phenotype can be obtained if treatment is begun in the symptomatic phase.

\section{Methods}

Further information can be found in Supplemental Methods, available online with this article; https://doi.org/10.1172/JCI99417DS1.

siRNA synthesis. siRNAs targeting TGAGAACAAAAATTGGGTTTTAA in hAGT (NM_00029.3) and GAGCTATCCTGGGCAAAAATCAA in rAGT (NM_134432.2) were prepared as chemically modified triantennary GalNAc conjugates as described previously (33). Duplexes were synthesized on controlled pore glass (CPG) solid support on a MerMade 192 synthesizer at $1 \mu \mathrm{mol}$ scale. The synthesis of $A G T$ sequences was performed using solid-supported oligonucleotide methodology and standard phosphoramidite chemistry. Amidite solutions were prepared at $0.1 \mathrm{M}$ concentration, and ethyl thiotetrazole (0.6 $\mathrm{M}$ in acetonitrile) was used as activator. Deblock solution (3\% dichloroacetic acid in dichloromethane), oxidizer solution (50 mM iodine in 9:1 pyridine/water mixture), and capping reagent $(A+B)$ solutions were prepared according to reported procedures (68). Sense and antisense single strands were chemically modified at selected sites with either 2'-O-methyl or 2'-F nucleosides. Sense strand was synthesized on a solid support loaded with a triantennary GalNAc moiety, while the antisense sequence was synthesized on a solid support loaded with 2'-OMe A.

The synthesized sequences were cleaved and deprotected in 96-well plates, using aqueous methylamine reagent (25-45 minutes on column to cleave the sequences from the solid support, filtered, followed by continued incubation of the crude oligonucleotide filtrate at $60^{\circ} \mathrm{C}$ for 30 minutes). After centrifugation and decanting of the solvents, the pellet was isolated and resuspended in $0.2 \mathrm{M}$ sodium acetate buffer. The single strands were analyzed by liquid chromatographymass spectrometry to confirm the identity, by UV for quantification, and by ion exchange chromatography to determine purity.

Purification and desalting. Resuspended Agt single strands were desalted using an AKTA Purifier system equipped with a Sephadex G25 column. The process was run at ambient temperature. Sample injection and collection were performed in 96-well plates $(1.8-\mathrm{mL}$ deep well plates). A single peak for each sequence was collected in the eluent. The desalted $A G T$ sequences were analyzed for concentration (by UV measurement at A260) and purity (by ion exchange HPLC). The complementary single strands were then combined in a 1:1 stoichiometric ratio to form siRNA duplexes.

Animals. Female Sprague-Dawley rats harboring the hAgt gene [TGR(hAogen)L1623] were mated with male Sprague-Dawley rats bearing the human renin (hRen) gene [TGR(hRen)L10J] to produce a model of preeclampsia (PE) in the dams (69). These pregnant dams develop hypertension on day 13 of pregnancy (plug-recognition day is assigned as day 1) and albuminuria (26). Mean blood pressure was continuously recorded by radiotelemetry. The implantation of radiotelemetry pressure transducers (TA11PA-C20, Data Sciences International, La Jolla, California, USA) was performed 10 days before mating as described previously (26). Twenty-four-hour urine samples were collected in metabolic cages at day 17/18 of gestation.

Transgenic dams were randomly assigned to 2 experimental groups: hAgt-targeting siRNA or luciferase-targeting siRNA as control. Beginning on day 3 of gestation, transgenic hAgt dams were dosed s.c. with $10 \mathrm{mg} / \mathrm{kg}$ siRNA every third day through gestation day 15. This regimen was chosen to maximize exposure, for assessment of fetal effects and exposure. 
The RUPP procedure is a well-established model for studying the link between placental ischemia and hypertension in the pregnant rat and has been described in detail previously (70). First timed pregnant Sprague-Dawley rats purchased from Harlan Sprague-Dawley Inc. were used in this study. Twenty-eight pregnant rats were randomly assigned to the NP, NP rAgt-targeting siRNA, RUPP, or RUPP rAgt-targeting siRNA group. On day 12 of gestation, siRNA was injected s.c. into rats (10 $\mathrm{mg} / \mathrm{kg}$ ). On gestational day 14 , under isoflurane anesthesia, NP rats assigned to one of the RUPP groups underwent a reduction in uterine perfusion pressure (RUPP) (described in detail in Supplemental Methods). Animals were instrumented, and arterial pressure was determined in all groups of rats at day 19 of gestation as described previously (70).

All experimental animals were sacrificed on day 19 (RUPP model) or 21 (transgenic model). The fetuses and organs were removed, weighed, and collected. All surgery was performed under isoflurane anesthesia, and all efforts were made to minimize suffering.

Urinary rat albumin was measured with a commercially available ELISA (CellTrend). Serum cystatin C was measured with a commercially available ELISA (BioVendor), and serum creatinine was determined by an automated clinical method. Serum PLGF (R\&D Systems) and sFLT (MyBiosource) concentrations were measured with a commercially available ELISA. Plasma $\mathrm{PGE}_{2}$ concentrations were measured with a commercially available ELISA (R\&D Systems).

siRNA quantification assay. Maternal liver, fetal liver, and placenta levels of hAGT-targeting siRNA were quantified via stem loop quantitative reverse transcriptase PCR (qRT-PCR). In short, aliquots of the tissues (frozen, powderized) were reconstituted to $100 \mathrm{mg} / \mathrm{mL}$ in PBS with $0.25 \%$ Triton X-100 and lysed by boiling. Further dilution of samples on a naive background matrix was made when the concentration of the siRNA might be expected to exceed the upper limit of quantification. The obtained supernatant subsequently was used to generate antisense-specific cDNA using a sequence-specific stem loop cDNA primer (GTCGTATCCAGTGCAGGGTCCGAGGTATTCGCACTGGATACGACtgagaa).

Antisense strand levels were quantified using a sequence-specific TaqMan assay and LightCycler 480 system (Roche). The TaqMan assay components were a forward primer (GCTGTTAAAACCCAATTTTTGT), reverse primer (GTGCAGGGTCCGAGGT), and probe (6FAM-TCCGAGGTA/ZEN/TTCGCACTGGAT-Iowa Black FQ). siRNA concentration was determined by interpolation from the standard curves generated by spiking of various concentrations of the siRNA in the naive tissue and processed as described above. The lower limit of quantitation, defined as 2 standard deviations above background, was $1.3 \mathrm{ng} / \mathrm{g}$ tissue.

Measurement of AGT and angiotensin II levels. hAGT and rAGT as well as angiotensin (Ang) peptide levels in the serum were measured by Attoquant Diagnostics $\mathrm{GmbH}$, using mass spectrometry to investigate Ang metabolites. This technique allows for measurement of 10 metabolites, including Ang 1-10 (Ang I), Ang 1-8 (Ang II), Ang 1-7 (MAS receptor agonist), Ang 2-8 (Ang III), and Ang 3-8 (Ang IV). Samples were spiked with 100 pg/mL stableisotope-labeled internal standards and subjected to solid-phase extraction using Sep-Pak cartridges (Waters) according to the manufacturer's protocol. After elution and solvent evaporation, samples were reconstituted in $50 \mu \mathrm{L} 50 \%$ acetonitrile/0.1\% formic acid and subjected to liquid chromatography-tandem mass spectrometry analysis using a reversed-phase analytical column (Luna C18, Phenomenex) with a gradient ranging from $10 \%$ acetonitrile/0.1\% formic acid to $70 \%$ acetonitrile/0.1\% formic acid in 9 minutes. The eluate was analyzed in line with a QTRAP-4000 mass spectrometer (AB Sciex) operated in the MRM mode using dwell times of 25 milliseconds at a cone voltage of $4000 \mathrm{~V}$ and a source temperature of $300^{\circ} \mathrm{C}$. For each peptide and corresponding internal standards, 2 different mass transitions were measured. Ang II peptide concentrations were calculated by relating of endogenous peptide signals to internal standard signals.

mRNA isolation and real-time RT-PCR. Total mRNA was isolated using QIAzol lysis reagent and Qiagen RNeasy Mini Kit (Qiagen) with on-column deoxyribonuclease I step (Qiagen) according to the manufacturer's protocol. Two micrograms of mRNA was reverse-transcribed into cDNA using a High Capacity cDNA Reverse Transcription Kit (Applied Biosystems). Real-time PCR was detected on an ABI 7500 Fast Sequence Detection System (Applied Biosystems) and analyzed by 7500 Fast System Software (Applied Biosystems). Primers and probes (Supplemental Table 1) were designed with PrimerExpress 3.0 (Applied Biosystems) and synthesized by Biotez.

Immunohistochemistry and histochemistry. Rat uteroplacental units were fixed in buffer as described earlier (26), truncated from 2 lateral placental parts, and embedded in paraffin. Consecutive sections were stained for a trophoblast cell marker (cytokeratin [clone MNF116]; M0821, DAKO), a smooth muscle cell marker ( $\alpha$-actin [clone 1A4]; M0851, DAKO), and CD68, a marker for monocytes and macrophages (clone ED1; MCA341R, AbD Serotec). Sections were visualized on a Zeiss Axio Imager M2 microscope fitted with an AxioCamHRc camera (Zeiss) using $\times 10$ magnification. On cross sections of the most central part of the uteroplacental unit, areas (placental area, mesometrial triangle area, and interstitial trophoblast cell invasion into mesometrial triangle) were calculated using AxioVision software (Zeiss). Actin staining was used to set the border between mesometrial triangle and myometrium. Interstitial trophoblast cell invasion into mesometrial triangle was calculated as the area of trophoblast cells in the mesometrial triangle. The number of macrophages was determined by image analysis using Image (NIH). Masson's trichrome staining of the uteroplacental unit was performed using standard protocols. The whole stained sections of the uteroplacental unit were imaged by a Pannoramic MIDI II BF/FL high-speed slide scanner (3DHISTECH Ltd.), saved, and evaluated offline in a blinded manner using CaseViewer analysis software (3DHISTECH Ltd.). We analyzed 9 uteroplacental unit sections of each group from 6 luciferase-targeting siRNA-treated $\mathrm{PE}$ rats and $9 h A G T$-targeting siRNA-treated PE rats at gestational day 21. On average, there were 57 vessels in the luciferase-targeting siRNAtreated group and 97 vessels in the $h A G T$-targeting siRNA-treated group. For the interpretation of vascular remodeling of spiral arteries, the outer diameter (OD) and luminal diameter (LD) of each vessel were measured at the point of the largest OD using a Pannoramic Viewer (3DHISTECH Ltd.). The ratio of LD to OD was used to compare the proportionate vessel wall thickness of the spiral arteries in the mesometrial triangle. Masson's trichrome and H\&E stainings of the kidney sections were performed using standard protocols. For the interpretation of renal morphology, the number of glomeruli was counted, and fibrosis, tubular dysgenesis, and glomerular endotheliosis were evaluated using a Pannoramic Viewer (3DHISTECH Ltd.). H\&E staining of the lung sections was performed using standard protocols. To measure the mean linear intercept (Lm), as an index of alveolar size, we did a simple point and intersection counting in images of H\&E-stained lung sections of 3-week-old offspring using a suitable coherent test line system with unit length $d$ - that is, counting points hitting airspaces, $P$ (airspace), and intersections of a test line system with alveolar surface, $I(\mathrm{a})$, to obtain $\mathrm{Lm}=2 \times d \times P($ airspace $) / I(\mathrm{a})(71)$. 
Fetal and juvenile brain volumetric analysis. Brain volume analysis was performed as described previously $(72,73)$. In brief, fetal (embryonic day 21) and juvenile (postnatal day 21) rat brains were fixed in $4 \%$ formalin and embedded in paraffin. To determine brain volumes, $10-\mu \mathrm{m}$-thick paraffin sections (every $160 \mu \mathrm{m}$ between +1.54 and $-4.08 \mathrm{~mm}$ from bregma according to Paxinos and Watson, ref. 74) were stained with cresyl violet. Sections were scanned with a slide scanner (OpticLab H850, PlusTek). The area of each section was analyzed using Image J software (NIH), and volumetric analysis was performed by integration of the areas.

$\mu C T$ of fetal skulls. Six embryo heads per group were imaged in a SkyScan $1276 \mu \mathrm{CT}$ scanner (Bruker). The following acquisition parameters were used: source voltage, $55 \mathrm{kV}$; source current, 200 $\mu \mathrm{A}$; exposure time, $408 \mathrm{~ms}$; $\mathrm{Al} 0.5-\mathrm{mm}$ filter; $360^{\circ}$ rotation with a rotation step of $0.4^{\circ}$; and 2 averages. The images were reconstructed with NRecon software (Bruker) applying the beam-hardening correction of $28 \%$ and ring-artifact correction of 37 . The width and length of the embryo skull were measured.

Preparation of cardiomyocytes and cardiomyocyte beating rate assay. Isolation and cultivation of neonatal heart cells were performed as described previously (15). Immunoglobulin was isolated from $200 \mu \mathrm{L}$ of rat serum by protein $\mathrm{G}$-Sepharose on a Bioline protein purification system (Knauer). This IgG fraction was used in the bioassay. AT1-AA activity was measured using spontaneously beating neonatal rat cardiomyocytes and characterized and antagonized specifically using the AT1 antagonist losartan. Standardization and measurements were described in more detail previously (15).

Statistics. All data are presented as means \pm SEM. Normal distribution was assessed by Kolmogorov-Smirnov test. Group differences were analyzed by 2-tailed $t$ test, Mann-Whitney $U$ test, 1-way
ANOVA with Tukey's post hoc test, or 2-way ANOVA with Bonferroni's post hoc test, as appropriate. $P$ less than 0.05 was considered statistically significant.

Study approval. Local authorities approved the studies (State Office for Health and Social Affairs [LaGeSo], Berlin, Germany, permit G0095/15; and IACUC, University of Mississippi, Jackson, Mississippi, USA), which were performed according to American Physiological Society guidelines.

\section{Author contributions}

$\mathrm{NH}$ designed research studies, conducted experiments, acquired data, analyzed data, and wrote the manuscript. DJF designed research studies, identified siRNAs and dosing regimens, and wrote the manuscript. MWC, JB, and GW conducted experiments and acquired data. SM, TN, and SSM quantified siRNA in tissue. SS and JR synthesized siRNA and provided siRNA. MG, KK, FH, MS, IB, $\mathrm{HN}, \mathrm{AH}, \mathrm{AG}$, and $\mathrm{KT}$ analyzed data. BL designed research studies and supervised experiments. DNM and RD designed research studies, supervised experiments, and wrote the manuscript.

\section{Acknowledgments}

This work was supported by Alnylam Pharmaceuticals (Cambridge, Massachusetts, USA). We thank Jutta Meisel, Astrid Schiche, Juliane Anders, and Ute Gerhard for their excellent technical assistance.

Address correspondence to: Don Foster, Alnylam Pharmaceuticals, 300 Third Street, Cambridge, Massachusetts 02142, USA. Phone: 617.682.4052; Email: dfoster@alnylam.com.
1. Steegers EA, von Dadelszen P, Duvekot JJ, Pijnenborg R. Pre-eclampsia. Lancet. 2010;376(9741):631-644.

2. American College of Obstetricians Gynecologists, Task Force on Hypertension in Pregnancy. Hypertension in pregnancy. Report of the American College of Obstetricians and Gynecologists' Task Force on Hypertension in Pregnancy. Obstet Gynecol. 2013;122(5):1122-1131.

3. Barker DJ, Fall CH. Fetal and infant origins of cardiovascular disease. Arch Dis Child. 1993;68(6):797-799.

4. Irgens HU, Reisaeter L, Irgens LM, Lie RT. Long term mortality of mothers and fathers after pre-eclampsia: population based cohort study. BMJ. 2001;323(7323):1213-1217.

5. Irani RA, Xia Y. Renin angiotensin signaling in normal pregnancy and preeclampsia. Semin Nephrol. 2011;31(1):47-58.

6. Paulis L, Unger T. Novel therapeutic targets for hypertension. Nat Rev Cardiol. 2010;7(8):431-441.

7. Jeunemaitre X, et al. Molecular basis of human hypertension: role of angiotensinogen. Cell. 1992;71(1):169-180.

8. Johnson $\mathrm{AD}$, et al. Association of hypertension drug target genes with blood pressure and hypertension in 86,588 individuals. Hypertension. 2011;57(5):903-910.

9. Olearczyk J, et al. Targeting of hepatic angiotensinogen using chemically modified siRNAs results in significant and sustained blood pres- sure lowering in a rat model of hypertension. Hypertens Res. 2014;37(5):405-412.

10. Stec DE, Davisson RL, Haskell RE, Davidson BL, Sigmund CD. Efficient liver-specific deletion of a floxed human angiotensinogen transgene by adenoviral delivery of Cre recombinase in vivo. J Biol Chem. 1999;274(30):21285-21290.

11. $\mathrm{Wu} \mathrm{CH}$, et al. Adipocyte (pro)renin-receptor deficiency induces lipodystrophy, liver steatosis and increases blood pressure in male mice. Hypertension. 2016;68(1):213-219.

12. Gant NF, Daley GL, Chand S, Whalley PJ, MacDonald PC. A study of angiotensin II pressor response throughout primigravid pregnancy. J Clin Invest. 1973;52(11):2682-2689.

13. Mistry HD, Kurlak LO, Broughton Pipkin F. The placental renin-angiotensin system and oxidative stress in pre-eclampsia. Placenta. 2013;34(2):182-186.

14. AbdAlla S, Lother H, el Massiery A, Quitterer U. Increased AT(1) receptor heterodimers in preeclampsia mediate enhanced angiotensin II responsiveness. Nat Med. 2001;7(9):1003-1009.

15. Dechend R, et al. Agonistic autoantibodies to the AT1 receptor in a transgenic rat model of preeclampsia. Hypertension. 2005;45(4):742-746.

16. Ward K, et al. A molecular variant of angiotensinogen associated with preeclampsia. Nat Genet. 1993;4(1):59-61.

17. Inoue I, et al. A mutation of angiotensinogen in a patient with preeclampsia leads to altered kinet- ics of the renin-angiotensin system. J Biol Chem. 1995;270(19):11430-11436.

18. Wenzel K, et al. Angiotensin II type 1 receptor antibodies and increased angiotensin II sensitivity in pregnant rats. Hypertension. 2011;58(1):77-84.

19. Alexander BT, Cockrell K, Cline FD, Llinas MT, Sedeek M, Granger JP. Effect of angiotensin II synthesis blockade on the hypertensive response to chronic reductions in uterine perfusion pressure in pregnant rats. Hypertension. 2001;38(3 pt 2):742-745.

20. Brewer J, et al. Endothelin-1, oxidative stress, and endogenous angiotensin II: mechanisms of angiotensin II type I receptor autoantibody-enhanced renal and blood pressure response during pregnancy. Hypertension. 2013;62(5):886-892.

21. Buttar HS. An overview of the influence of ACE inhibitors on fetal-placental circulation and perinatal development. Mol Cell Biochem. 1997;176(1-2):61-71.

22. Burrows RF, Burrows EA. Assessing the teratogenic potential of angiotensin-converting enzyme inhibitors in pregnancy. Aust NZ J Obstet Gynaecol. 1998;38(3):306-311.

23. von Dadelszen P, Ornstein MP, Bull SB, Logan AG, Koren G, Magee LA. Fall in mean arterial pressure and fetal growth restriction in pregnancy hypertension: a meta-analysis. Lancet. 2000;355(9198):87-92.

24. Magee LA, et al. Less-tight versus tight control of hypertension in pregnancy. N Engl J Med. 
2015;372(5):407-417.

25. Takimoto E, Ishida J, Sugiyama F, Horiguchi H, Murakami K, Fukamizu A. Hypertension induced in pregnant mice by placental renin and maternal angiotensinogen. Science. 1996;274(5289):995-998.

26. Hering L, et al. Effects of circulating and local uteroplacental angiotensin II in rat pregnancy. Hypertension. 2010;56(2):311-318.

27. Anton L, Brosnihan KB. Systemic and uteroplacental renin-angiotensin system in normal and pre-eclamptic pregnancies. Ther Adv Cardiovasc Dis. 2008;2(5):349-362.

28. Li J, LaMarca B, Reckelhoff JF. A model of preeclampsia in rats: the reduced uterine perfusion pressure (RUPP) model. Am J Physiol Heart Circ Physiol. 2012;303(1):H1-H8.

29. Elbashir SM, Harborth J, Lendeckel W, Yalcin A, Weber K, Tuschl T. Duplexes of 21-nucleotide RNAs mediate RNA interference in cultured mammalian cells. Nature. 2001;411(6836):494-498.

30. Bobbin ML, Rossi JJ. RNA Interference (RNAi)based therapeutics: delivering on the promise? Annu Rev Pharmacol Toxicol. 2016;56:103-122.

31. Fitzgerald K, Kallend D, Simon A. A highly durable RNAi therapeutic inhibitor of PCSK9. N Engl JMed. 2017;376(18):e38.

32. Nair JK, et al. Multivalent N-acetylgalactosamine-conjugated siRNA localizes in hepatocytes and elicits robust RNAimediated gene silencing. J Am Chem Soc. 2014;136(49):16958-16961.

33. Matsuda S, et al. siRNA conjugates carrying sequentially assembled trivalent $\mathrm{N}$-acetylgalactosamine linked through nucleosides elicit robust gene silencing in vivo in hepatocytes. ACS Chem Biol. 2015;10(5):1181-1187.

34. Rana S, Karumanchi SA, Lindheimer MD. Angiogenic factors in diagnosis, management, and research in preeclampsia. Hypertension. 2014;63(2):198-202.

35. Hoeber D, et al. Erythropoietin restores longterm neurocognitive function involving mechanisms of neuronal plasticity in a model of hyperoxia-induced preterm brain injury. Oxid Med Cell Longev. 2016;2016:9247493.

36. Endesfelder S, Weichelt U, Schiller C, Sifringer M, Bendix I, Bührer C. Caffeine protects against anticonvulsant-induced neurotoxicity in the developing rat brain. Neurotox Res. 2017;32(3):460-472.

37. Krause M, Rak-Raszewska A, Pietilä I, Quaggin SE, Vainio $S$. Signaling during kidney development. Cells. 2015;4(2):112-132.

38. Mari C, Winyard P. Concise Review: Understanding the renal progenitor cell niche in vivo to recapitulate nephrogenesis in vitro. Stem Cells Transl Med. 2015;4(12):1463-1471.

39. Clyman RI, Mauray F, Roman C, Rudolph AM, Heymann MA. Circulating prostaglandin E2 concentrations and patent ductus arteriosus in fetal and neonatal lambs. JPediatr. 1980;97(3):455-461.

40. Clyman RI. Mechanisms regulating the ductus arteriosus. Biol Neonate. 2006;89(4):330-335.

41. Stoller JZ, Demauro SB, Dagle JM, Reese J. Cur- rent perspectives on pathobiology of the ductus arteriosus. J Clin Exp Cardiolog. 2012;8(1):S8-001.

42. Uijl E, et al. Strong and sustained antihypertensive effect of small interfering RNA targeting liver angiotensinogen. Hypertension. 2019;73(6):1249-1257.

43. Zlatev I, et al. Reversal of siRNA-mediated gene silencing in vivo. Nat Biotechnol. 2018;36(6):509-511.

44. Wallukat $\mathrm{G}$, et al. Patients with preeclampsia develop agonistic autoantibodies against the angiotensin AT1 receptor. JClin Invest. 1999;103(7):945-952.

45. Zhou CC, et al. Angiotensin receptor agonistic autoantibodies induce pre-eclampsia in pregnant mice. Nat Med. 2008;14(8):855-862.

46. Maynard SE, et al. Excess placental soluble fms-like tyrosine kinase 1 (sFlt1) may contribute to endothelial dysfunction, hypertension, and proteinuria in preeclampsia. JClin Invest. 2003;111(5):649-658.

47. Vander AJ, Geelhoed GW. Inhibition of renin secretion by angiotensin. II. Proc Soc Exp Biol Med.1965;120(2):399-403.

48. Firth JA, Leach L. Not trophoblast alone: a review of the contribution of the fetal microvasculature to transplacental exchange. Placenta. 1996;17(2-3):89-96.

49. Petell JK, et al. Alteration in the regulation of plasma membrane glycoproteins of the hepatocyte during ontogeny. Exp Cell Res. 1990;187(2):299-308.

50. Yoshida S, Furuhashi M, Suganuma N. Expression of asialoglycoprotein receptor in human fetal liver. Endocr J. 1999;46(1):67-73.

51. Faas MM, Spaans F, De Vos P. Monocytes and macrophages in pregnancy and pre-eclampsia. Front Immunol. 2014;5:298.

52. Bulmer JN, Williams PJ, Lash GE. Immune cells in the placental bed. Int J Dev Biol. 2010;54(23):281-294.

53. Wenzel K, et al. Angiotensin II type 1 receptor antibodies and increased angiotensin II sensitivity in pregnant rats. Hypertension. 2011;58(1):77-84.

54. Herse F, et al. Dysregulation of the circulating and tissue-based renin-angiotensin system in preeclampsia. Hypertension. 2007;49(3):604-611.

55. Campbell N, LaMarca B, Cunningham MW. The role of agonistic autoantibodies to the angiotensin II type 1 receptor (AT1-AA) in pathophysiology of preeclampsia. Curr Pharm Biotechnol. 2018;19(10):781-785.

56. Dechend R, et al. AT1 receptor agonistic antibodies from preeclamptic patients stimulate $\mathrm{NADPH}$ oxidase. Circulation. 2003;107(12):1632-1639.

57. Dechend R, et al. AT(1) receptor agonistic antibodies from preeclamptic patients cause vascular cells to express tissue factor. Circulation. 2000;101(20):2382-2387.

58. Brewer J, et al. Endothelin-1, oxidative stress, and endogenous angiotensin II: mechanisms of angiotensin II type I receptor autoantibody-enhanced renal and blood pressure response during pregnancy. Hypertension. 2013;62(5):886-892.

59. Irani RA, et al. Autoantibody-mediated angio- tensin receptor activation contributes to preeclampsia through tumor necrosis factor-alpha signaling. Hypertension. 2010;55(5):1246-1253.

60. Bian J, et al. Limited AT1 receptor internalization is a novel mechanism underlying sustained vasoconstriction induced by AT1 receptor autoantibody from preeclampsia. JAm Heart Assoc. 2019;8(6):e011179.

61. Murphy SR, Cockrell K. Regulation of soluble fms-like tyrosine kinase-1 production in response to placental ischemia/hypoxia: role of angiotensin II. Physiol Rep. 2015;3(2):e12310.

62. Forrester SJ, et al. Angiotensin II signal transduction: an update on mechanisms of physiology and pathophysiology. Physiol Rev. 2018;98(3):1627-1738.

63. Kanasty R, Dorkin JR, Vegas A, Anderson D. Delivery materials for siRNA therapeutics. Nat Mater. 2013;12(11):967-977.

64. Fitzgerald K, et al. Effect of an RNA interference drug on the synthesis of proprotein convertase subtilisin/kexin type 9 (PCSK9) and the concentration of serum LDL cholesterol in healthy volunteers: a randomised, single-blind, placebo-controlled, phase 1 trial. Lancet. 2014;383(9911):60-68.

65. Coelho T, et al. Safety and efficacy of RNAi therapy for transthyretin amyloidosis. $N$ EnglJ Med. 2013;369(9):819-829.

66. Thi EP, et al. Lipid nanoparticle siRNA treatment of Ebola-virus-Makona-infected nonhuman primates. Nature. 2015;521(7552):362-365.

67. Mehta PK, Griendling KK. Angiotensin II cell signaling: physiological and pathological effects in the cardiovascular system. Am J Physiol Cell Physiol. 2007;292(1):C82-C97.

68. Beaucage SL. Solid-phase synthesis of siRNA oligonucleotides. Curr Opin Drug Discov Devel. 2008;11(2):203-216.

69. Bohlender J, Ganten D, Luft FC. Rats transgenic for human renin and human angiotensinogen as a model for gestational hypertension. J Am Soc Nephrol. 2000;11(11):2056-2061.

70. Granger JP, et al. Reduced uterine perfusion pressure (RUPP) model for studying cardiovascular-renal dysfunction in response to placental ischemia. Methods Mol Med. 2006;122:383-392.

71. Hsia CC, Hyde DM, Ochs M, Weibel ER, ATS/ERS Joint Task Force on Quantitative Assessment of Lung Structure. An official research policy statement of the American Thoracic Society/European Respiratory Society: standards for quantitative assessment of lung structure. Am J Respir Crit Care Med. 2010;181(4):394-418.

72. Golic M, et al. Diabetes mellitus in pregnancy leads to growth restriction and epigenetic modification of the Srebf 2 gene in rat fetuses. Hypertension. 2018;71(5):911-920.

73. Przybyl L, et al. Regulatory T cells ameliorate intrauterine growth retardation in a transgenic rat model for preeclampsia. Hypertension. 2015;65(6):1298-1306.

74. Paxinos G, Watson C. The Rat Brain in Stereotaxic Coordinates. Cambridge, Massachusetts, USA Elsevier; 1996. 